\title{
Thalamocortical Response Transformations in Simulated Whisker Barrels
}

\author{
Harold T. Kyriazi and Daniel J. Simons \\ Department of Physiology, University of Pittsburgh School of Medicine, Pittsburgh, Pennsylvania 15261
}

Layer IV of rodent somatosensory cortex contains identifiable networks of neurons, called "barrels," that are related one-to-one to individual whiskers on the face. A previous study (Simons and Carvell, 1989) described differences between the response properties of thalamic and cortical vibrissa neurons and proposed that these transformations can be explained by several features of barrel anatomy and physiology: nonlinear neuronal properties, strongly responsive inhibitory and less responsive excitatory neurons, convergent thalamic inputs to cells of both types, and interconnections among barrel neurons. In the present study these features were incorporated into a computational model in order to test their explanatory power quantitatively. The relative numbers of excitatory and inhibitory cells and the relative numbers of synapses of thalamic and intrabarrel origin were chosen to be consistent with available light and electron microscopic data. Known functional differences between excitatory and inhibitory barrel neurons were simulated through differences in spike activation functions, refractory periods, postsynaptic potential decay rates, and synaptic strengths. The model network was activated by spike trains recorded previously from thalamic neurons in response to three different whisker deflection protocols, and output, which consisted of spikes generated by the simulated neurons, was compared to data from our previous neurophysiological experiments. For each type of whisker stimulus, the same set of parameter values yielded accurate simulations of the cortical response. Realistic output was obtained under conditions where each barrel cell integrated excitatory and inhibitory synaptic inputs from a number of thalamic and other barrel neurons and where the ratios between network excitation, network inhibition, and thalamic excitation were approximately constant. Several quantities are defined that may be generally useful in characterizing neuronal networks. One important implication of the results is that thalamic relay neurons not only provide essential drive to the cortex but could, by changing their tonic activities, also directly regulate the tonic inhibition present in the cortex and thereby modulate cortical receptive field properties.

[Key words: neural circuit, neuronal network, somatosensory cortex, computer model, receptive field, inhibition]

\footnotetext{
Received Mar. 27, 1992; revised Sept. 28, 1992; accepted Oct. 14, 1992.

We thank Paul Munro for advice on an early version of this model and George Carvell and G. Bard Ermentrout for useful comments and suggestions. This research was supported by NIH Grant NS19950 and by a grant from the Pittsburgh Supercomputing Center through the NIH Division of Research Resources Cooperative Agreement U41 RR04154.

Correspondence should be addressed to Harold Kyriazi at the above address.

Copyright (C) 1993 Society for Neuroscience $0270-6474 / 93 / 131601-15 \$ 05.00 / 0$
}

In sensory and other areas of the cerebral neocortex, neighboring neurons are thought to comprise neuronal assemblies in which afferent information from the thalamus is processed (Mountcastle, 1979). Such assemblies can be readily visualized in the face area of the rodent primary somatosensory cortex, where aggregations of layer IV neurons, called barrels, are related oneto-one to individual vibrissae on the contralateral mystacial pad (Woolsey and Van der Loos, 1970). Each barrel is organized with respect to a segregated input of thalamocortical (TC) axons whose terminal arbors in layer IV are confined almost exclusively to the barrel's center (Bernardo and Woolsey, 1987; Jensen and Killackey, 1987). These projections arise from the homologous ventrobasal (VB) thalamic barreloid and constitute the barrel's predominant source of extrinsic inputs (Land et al., 1986; Koralek et al., 1988). The barrels contain two major cell classes corresponding to spiny stellate cells and smooth nonpyramidal cells that have been described in different cortical areas in a variety of species (Woolsey et al., 1975; Simons and Woolsey, 1984). The dendritic fields of barrel neurons are confined to the barrel, and their axons ramify extensively within it where they come into close apposition to barrel neurons of both classes (Harris and Woolsey, 1983). Since thalamic contacts account for only $20 \%$ of all barrel synapses (White, 1978) and since locally projecting axons of non-barrel neurons largely avoid barrel centers (Bernardo et al., 1990a,b), it appears that a majority of synaptic connections in a barrel arise from neurons within it. Thus, available evidence strongly indicates that a barrel is a densely interconnected network of layer IV neurons that receives its predominant afferent drive from a single external source.

A recent physiological study (Simons and Carvell, 1989) compared response properties of the three principal cell types that comprise the barreloid/barrel circuit: thalamocortical units (TCUs), regular-spike barrel units (RSUs), and fast-spike barrel units (FSUs). Of the cortical cells, the former are thought to correspond to spiny neurons and the latter to smooth cells, which are GABAergic and inhibitory (Simons and Woolsey, 1984; McCormick et al., 1985). Functionally, RSUs differ from TCUs in three important respects. First, RSUs have much less spontaneous activity but only slightly smaller stimulus-evoked responses. Second, whereas both RSUs and TCUs respond maximally to deflections of a particular vibrissa, defined as the principal whisker (PW), the latter are more likely to respond strongly also to deflections of adjacent whiskers. Third, for RSUs, deflection of an adjacent whisker $20 \mathrm{msec}$ prior to deflection of the PW consistently suppresses the PW response, whereas such "cross-whisker" inhibition is observed much less frequently for TCUs. Thus, compared to TCUs, RSUs display a greater signalto-noise ratio, have more tightly focused excitatory receptive fields, and have stronger inhibitory surrounds. By contrast, FSUs 
respond most vigorously to whisker stimuli, and they have the greatest spontaneous activities and the largest receptive fields (see also Swadlow, 1989).

Simons and Carvell (1989) proposed that the greater overall responsiveness of FSUs relative to RSUs reflects intrinsic biophysical differences between smooth and spiny barrel cells (McCormick et al., 1985; Agmon and Connors, 1992) and differences in the pattern of their TC synapses. The former receive the bulk of their thalamic inputs on proximal dendritic shafts and somata, whereas the latter receive thalamic synapses on their spines, scattered along the length of the dendrite (White, 1978, 1979), and more proximal inputs may be stronger (Rall, 1967; Tsukahara et al., 1975). Spiny cell/RSUs presumably are depolarized to threshold only by the most numerous and synchronously active thalamic inputs, such as those engaged by deflecting the PW, thus accounting for their improved signalto-noise ratios and more tightly focused receptive fields. Conversely, smooth cell/FSUs more faithfully reflect activity in thalamic afferents, responding vigorously to stimulation of both principal and adjacent whiskers. Responses evoked by the latter can produce cross-whisker inhibition by means of connections from smooth cell/FSUs to spiny cell/RSUs. The effectiveness of adjacent whisker inhibition is enhanced by the broadly focused excitatory receptive fields of the smooth cell/FSUs, which are thought to reflect convergent inputs from cells in the corresponding barreloid, many of which have multiwhisker receptive fields. Spiny cell/RSUs also receive convergent thalamic inputs but are disproportionately less responsive to adjacent whisker stimuli, which typically evoke EPSPs but not spikes (Carvell and Simons, 1988). TC convergence onto both cell types is thought to account also for the finding that both FSUs and RSUs respond well to more angular directions of whisker movement than TCUS. As in the case of receptive field size, the greater overall responsiveness of the smooth cell/FSUs renders them responsive to more deflection angles than spiny cell/RSUs.

This biological model is based on four principal features of barrel organization: nonlinear neuronal properties, differential responsiveness of spiny and smooth barrel neurons, convergent thalamic inputs to cells of both types, and interconnections among neurons within a barrel. These features are consistent with some general rules of cortical organization that have been defined by White in a comprehensive review of cortical circuitry (White, 1989). In the present study we incorporated these features into a computational model of a barrel-like neuronal network in order to explore how they might contribute to the aforementioned TC response transformations in the whisker/barrel system. Our intent was to capture the net effects of spatial and temporal integration that occur within a barrel, not to model a barrel in full anatomical and physiological detail. The neurons' dendritic processes were modeled as leaky, linear, voltage integrators whose proportions of thalamic and intrabarrel synaptic inputs were consistent with available light and electron microscopic data, and the neurons' spike initiation zones were modeled in a nonlinear and stochastic manner (see Johannesma and Van den Boogaard, 1985) in a way designed to produce a differential responsiveness of excitatory and inhibitory cells. Neurons in the simulated barrel were activated by prerecorded spike trains from real thalamic barreloid neurons in response to three different types of whisker stimuli. Results demonstrate that the model network can produce appropriate output for all whisker stimulation protocols examined, suggesting that it captures essential operational features of a real barrel.

\section{Materials and Methods}

\section{Model structure and anatomical constraints}

The model barrel consists of 100 neurons, 70 spiny/excitatory and 30 smooth/inhibitory, each of which receives a variable number of excitatory synapses from cells in the homologous thalamic barreloid. The choice of $30 \%$ inhibitory cells is a consensus figure derived from several anatomical studies using Golgi or immunohistochemical techniques (Woolsey et al., 1975; Simons and Woolsey, 1984; Lin et al., 1985; Spreafico et al., 1988). We estimate that these numbers correspond roughly to $2-5 \%$ of the real barrel cell populations (Lee and Woolsey, 1975; Pasternak and Woolsey, 1975; Curcio and Coleman, 1982; see also Welker and Woolsey, 1974). Connections among barrel cells were constrained as follows: a given cell cannot synapse on itself or make more than one synapse on any other cell; a given cell can contact more than one other cell (of either type), but all its synapses are of the same sign (all excitatory or all inhibitory); the sign and magnitude of a synapse remain constant during a simulation. The average number of thalamic cells that contact a given type of barrel neuron is called the thalamocortical (TC) convergence; TC convergences can be set differently for smooth and spiny cclls. The number of other barrel neurons contacted by a barrel neuron is termed its divergence, and like TC convergence, divergences also may differ for smooth and spiny cells. The network has no geometric organization; all modeled neurons have equal probabilities of contacting each other.

Synapses made by TC and spiny (Ex) neurons correspond to asymmetric and presumed excitatory synapses, whereas synapses made by smooth (In) cells correspond to symmetric and presumed inhibitory synapses. On average (see below), the model network contained 6540 synapses: TC, 1140; Ex, 4200; In, 1200. Estimates of the relative proportions of TC, Ex, and In synapses were based on tabulated data available from the quantitative electron microscopic studies of White and colleagues (White and Rock, 1980; White et al., 1984; Benshalom and White, 1986; Keller and White, 1987). Appropriate synaptic populations were achicved first by choosing TC convergence factors and hence the number of TC synapses in the barrel, and then by adjusting the numbers of synapses made by the spiny cell population to attain a network in which approximately one-fifth of all excitatory synapses were of TC origin. The average number of barrel neurons contacted by smooth cells was then adjusted to achieve an appropriate number of inhibitory synapses. Percentages of synapses in the simulated barrel and of estimated populations in real barrels are, respectively, TC, $17 \%$ and $18 \%$; Ex, $64 \%$ and $68 \%$; In, $18 \%$ and $15 \%$.

In order to simulate natural variability in divergences and in the proximal/distal locations of synapses, the number of synapses made by a given cell and synaptic strengths were assigned randomly, according to a Gaussian distribution, with only the mean and range values $( \pm 2.33$ standard deviations) being prespecified. In all cases but one, ranges were sct to bc half the mean; the exceptional case was the TC convergence onto smooth cells, whose variance was made large to reflect the marked heterogeneity that is known to exist in the numbers of TC synapses onto smooth barrel neurons (White et al., 1984; Keller and White, 1987).

\section{Model function and physiological constraints}

The operation of a model neuron is illustrated in Figure 1, and all model parameter values are listed in Table 1. Circuit dynamics involve spatial and temporal integration; each barrel neuron is modeled as a series of differential equations that summate synaptic inputs and compute the amount of decay of each type of postsynaptic potential (PSP) from one millisecond to the next. A PSP is generated if an action potential occurred previously in the presynaptic neuron, and the effectiveness of each synapse depends on its assigned weight, which represents PSP amplitude in millivolts.

Several guidelines were followed in assigning the relative strengths of different typcs of synapses. We used synaptic weight to represent proximal/distal locations of TC, Ex, and In synapses. Thalamocortical synapses tend to occur at proximal locations on smooth cells, whereas spiny cells receive their TC synapses almost exclusively on their spines, distributed along the length of their dendrites, which is consistent with the greater overall responsiveness of FSUs. This difference was modeled by assigning greater weights to TC synapses onto smooth cells. Spiny and smooth barrel neurons differ also with respect to the spatial distribution of non-TC synapses (White, 1989). For spiny cells, inhibitory synapses tend to be located proximally, on soma and dendritic shafts, whereas excitatory synapses are made more distally, on dendritic spines (White 


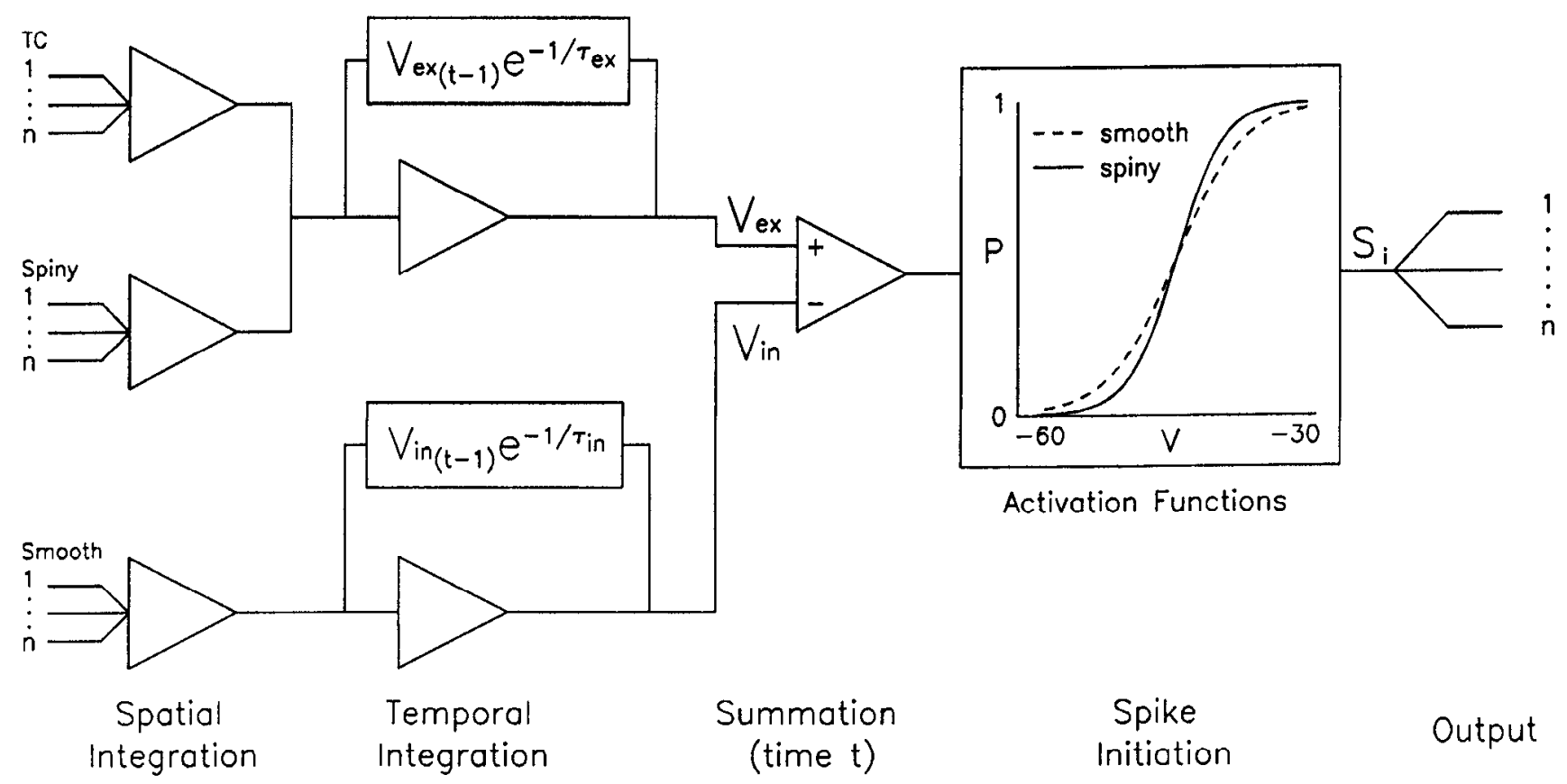

Figure 1. Operational diagram of a model barrel neuron. For spatial integration PSPs generated by all of the active excitatory synapses (TC and Spiny) and all of the active inhibitory synapses (Smooth) are summed separately. For temporal integration, PSP values are summed with residual PSPs remaining after their first-order temporal decay, where $\tau_{\text {ex }}$ and $\tau_{\text {in }}$ are the excitatory and inhibitory decay time constants. A net membrane potential $(V)$ is computed by summing depolarizing $(V$ ex $)$ and hyperpolarizing $(V$ in) PSP values, and this is used to determine the probability $(P)$ that an action potential occurs. If so, the cell's output $\left(S_{i}\right)$ can influence neurons postsynaptic to it. The shape of the activation function differs for smooth and spiny cells.

and Rock, 1980). Excitatory and inhibitory synapses onto smooth neurons tend to be more equally distributed along the entire length of the dendrites (Keller and White, 1987). On average, then, inhibitory synapses may be more effective in the case of spiny cells. We modeled this by assigning larger weights to the inhibitory synapses on the spiny cells. Aside from these general constraints, synaptic weights were unconstrained and were among the parameters varied most frequently in attempts to produce realistic output (see column 2 of Table 1). The synaptic weights listed in Table 1, particularly for TC synapses, are in the range of millivolts, not in the more physiological range of tenths of millivolts (see Mason et al., 1991). Such large PSPs werc necessitatcd in the model by its small size (see also Discussion) and by the fact that the model PSPs sum linearly whereas real thalamic inputs may not (Koch et al., 1983; Shepherd et al., 1985).

For each neuron, instantaneous net PSPs at time $t$ are computed by summing separately all of the PSPs from the set of active presynaptic TC, Ex, and In inputs. Excitatory postsynaptic potentials (EPSPs), which are generated by TC and Ex synapses, and inhibitory postsynaptic potentials (IPSPs), which are generated by synapses from smooth cells, are summed separately. EPSPs are depolarizing. IPSPs hyperpolarizing. The amplitude of each PSP rises instantaneously and decays first-order exponentially over time according to the following:

$$
V_{1}=V_{0} e^{-1 / \tau}
$$

where $V_{0}$ is the initial PSP valuc for cither Ex or In, $t$ is timc, and $\tau$ is the decay time constant. Each PSP contributes to a neuron's net activation for a time period determined by $\tau$. Since in vivo studies have demonstrated that the net response to a whisker stimulus is an initial excitation followed by a longer-lasting inhibition (Simons, 1985; Carvell

\section{Table 1. Model parameter values of best simulations}

\begin{tabular}{lllll} 
Parameter & $\Delta^{a}$ & VB & Spiny $^{b}$ & Smooth $^{b}$ \\
\hline Cell number & - & 100 & 70 & 30 \\
TC convergence to: & + & - & $12 \pm 6$ & $10 \pm 8$ \\
TC synaptic weight to: & +++ & - & $3.9 \pm 1.95 \mathrm{mV}$ & $6.0 \pm 3.0 \mathrm{mV}$ \\
Divergence from: & + & - & $60 \pm 30$ & $40 \pm 20$ \\
Spiny cell synaptic weight to: & +++ & - & $1.0 \pm 0.5 \mathrm{mV}$ & $1.0 \pm 0.5 \mathrm{mV}$ \\
Smooth cell synaptic weight to: & +++ & - & $2.05 \pm 1.025 \mathrm{mV}$ & $1.5 \pm 0.75 \mathrm{mV}$ \\
Refractory period & + & - & $4 \mathrm{msec}$ & $3 \mathrm{msec}$ \\
Resting membrane potential & - & - & $-60.0 \mathrm{mV}$ & $-60.0 \mathrm{mV}$ \\
Firing threshold & - & - & $-45.0 \mathrm{mV}$ & $-45.0 \mathrm{mV}$ \\
$\tau$ of PSPs evoked by: & ++ & $5 \mathrm{msec}$ & $5 \mathrm{msec}$ & $15 \mathrm{msec}$ \\
Threshold function temperature & +++ & - & 2.5 & 3.6 \\
Axon conduction times & - & $2 \mathrm{msec}$ & $1 \mathrm{msec}$ & $1 \mathrm{msec}$
\end{tabular}

${ }^{a}$ Denotes the extent to which effects of changes in parameter values were explored:,- never changed;,+ rarely;,++ occasionally; +++ , often.

${ }^{b} \pm$ denotes absolute ranges that correspond to $2.33 \mathrm{SDs}$ about the mean 
and Simons, 1988), EPSPs were assigned faster decay rates that IPSPs. Decay rates were otherwise unconstrained and adjusted empirically. Refractory periods for spiny and smooth cells were set at 4 and $3 \mathrm{msec}$, reflecting differences in the discharge capabilitics of thesc ncurons (Simons, 1978). Results equivalent to those presented herein can be obtained using an FSU refractory period of $2 \mathrm{msec}$, consistent with the limits defined in in vitro studies (McCormick et al., 1985), if, for example, a compensatory increase is made in the weight of inhibitory synapses onto smooth cells, along with minor adjustments in other parameters that affect smooth cell responsiveness (such as their EPSP and IPSP decay constants). During the refractory period action potentials were not allowed to occur, but PSPs were allowed to summate and decay normally; this ignores possible effects of the action potential on PSP integration in the dendrites. Also incorporated into the model are a $1.0 \mathrm{msec}$ synaptic delay for intrabarrel synapses $(0.5 \mathrm{msec}$ axon conduction time plus $0.5 \mathrm{msec}$ synaptic delay) and a $2.0 \mathrm{msec}$ delay for TC synapses ( $1.5 \mathrm{msec}$ axon conduction time plus $0.5 \mathrm{msec}$ synaptic delay). These values are generally consistent with results from cross-corrclation analyses of barrel neuron discharges (M. J. Bloom and D. J. Simons, unpublished observations) and from in vivo and in vitro studies of thalamocortical relations in the vibrissa system (Swadlow, 1989; Agmon and Connors, 1992). Thus, at time $t$ a PSP is generated at a synaptic site if an action potential occurred in the presynaptic element at time $t-2$ for TC synapses or $t-1$ for intracortical synapses.

The membrane potential of each cell is updated at $1.0 \mathrm{msec}$ intervals. It is the sum of all of the neuron's TC, Ex, and In inputs at time $t$ plus residual PSPs remaining after decay of previous inputs:

$$
\begin{aligned}
V_{(t)}= & \sum_{i=1}^{n} \operatorname{Ex}_{i}+\sum_{i=1}^{n} \mathrm{Tc}_{i}-\sum_{i=1}^{n} \operatorname{In}_{i}+V \operatorname{ex}_{(l-1)} e^{-1 / \tau_{\mathrm{cx}}} \\
& -V \operatorname{in}_{(i-1)} e^{-1 / \tau_{\text {in }} .}
\end{aligned}
$$

On the right-hand side of the equation, the first three terms constitute spatial summation and the last two terms, temporal integration.

The membrane potential is converted into a probability, whereupon a random number generator is used to determine whether or not the cell discharges an action potential. The activation function, which is taken from thermodynamic-type connectionist models (see Rumelhart et al., 1986), has the form

$$
P=1 /\left(1+e^{(\theta-V) / T}\right),
$$

where $P$ is the probability from 0.0 to $1.0, \theta$ is the firing threshold, $V$ is the membrane potential, and $T$ is the "temperature." Note that when $V=\theta, P=0.5$. Also, as tempcrature decreases, the slope of the activation function increases, more closely approximating a deterministic step function. Spiny cells were assigned a lower temperature than smooth cells, enhancing their nonlinearity (see Fig. 1).

The stochastic nature of the activation function allows the model neurons to fire spontaneously with low levels of spontaneous thalamic input activity, which in the model is asynchronous, a situation that may not be physiological (see Discussion). As illustrated in Figure 1, smooth cells have a much greater probability of spike discharge at low levels of depolarization. For spiny cells, the steep slope of the function near threshold exaggerates differences between relatively weak and strong inputs. At high levels of depolarization, differences in the functions for the smooth and spiny cells are largely irrelevant because probabilities of discharge are calculated every millisecond and the gradual decay of the PSPs makes it highly likcly that a cell depolarized beyond threshold will eventually fire. If a neuron generates a spike at time $t$, it influences cells postsynaptic to it at $t+1$, and remains refractory until $t+3$ for smooth and $t+4$ for spiny cells. For each cell, the times of occurrence of its action potentials were saved with a resolution of $1 \mathrm{msec}$.

\section{Sensory stimulation and thalamic input}

Whisker stimulation protocols. The model barrel was activated by prerecorded spike trains that had been obtained from thalamic barreloid neurons during our previous and ongoing studies of the whisker/barrel system. Simulations were conducted using three whisker stimulation protocols for which both thalamic and cortical data are available.

Initial simulations were conducted using input spike trains obtained by deflecting the PW alone. The whisker was moved in eight different directions, that is, in $45^{\circ}$ increments relative to the horizontal alignment of the whisker rows on the face. The whisker was moved rapidly to its deflected position and held there for approximately $200 \mathrm{msec}$ (see Simons and Carvell, 1989, for details). Hereafter, this is referred to as the "standard protocol." Data collection lasted for $500 \mathrm{msec}$, bracketing the whisker stimulus. Spike occurrences were saved as sequential interevent times. Each input data file consists of responses to 10 randomized sets of the eight stimulus directions (a total of 80 stimuli). Results are available from 242 RSUs and 16 FSUs. We estimate that a barreloid contains only $10-20 \%$ as many cells as its homologous barrel (Land et al., 1986). However, data files from 100 of the 135 thalamic neurons reported previously by Simons and Carvell (1989) were used as input to the model. Although this number represents considerably more than $10-20 \%$ of our modeled barrel's neuron population, we found it desirable to use as broad a sample of input units as possible. If the number of input neurons was made much smaller, to be more proportional to the total of 100 modeled barrel neurons, then the behavior of the model network could be strongly influenced by idiosyncratic spike patterns from only a handful of stimulus presentations.

After a set of model parameter values were found that produced realistic simulations of barrel neuron responses to the standard protocol, the same parameter values were used to test the network's ability to produce realistic barrel neuron responses to other stimulus conditions. One of the novel protocols was identical to the standard one except that the whisker was held in a deflected state for 1400 rather than $200 \mathrm{msec}$. As described below, use of such stimuli allowed us to evaluate temporal summation within the model network. Biologic data (Kyriazi and Simons, unpublished observations) were available for 65 TCUs, 68 RSUs, and 7 FSUs. The third stimulus protocol, which involved the sequential deflection of two whiskers in a condition-test paradigm (see Simons and Carvell, 1989), assessed two aspects of the spatial organization of whisker receptive fields: the excitatory responses evoked by whiskers surrounding the PW and the inhibition of PW responses by them. Deflection of an adjacent whisker at one of eight different deflection angles (the conditioning stimulus) occurred $20 \mathrm{msec}$ prior to deflection of the PW at its maximally effective angle (the conditioned test stimulus). The PW also was deflected alone (test stimulus alone). The protocol consists of 10 sets of nine stimuli, and for each cell up to four adjacent whiskers were tested. Data, which were from a subset of the cells studied with the standard protocol, included 21 TCUs, 21 RSUs, and 4 FSUs.

Treatment of the thalamic input. Each of the whisker stimulation protocols used randomized sequences of eight deflection angles. Since most thalamic and cortical neurons respond differently to whisker movements in different directions, there may be specificity in the connections between neurons in a thalamic barreloid and neurons in the homologous cortical barrel (for a discussion, see Lichtenstein et al., 1990). For all of the simulations, the assumption was made that barrel neurons receive their thalamic input from barreloid cells that respond maximally to the same (upward) direction of whisker movement. To simulate this, data in each thalamic input file were rotated so that all thalamic input neurons now responded as if their maximally effective stimulus was an upward deflection. Also, the presentation of the eight deflection angles was derandomized in order to mimic a real population of thalamic responses to a series of whisker deflections at specific angles.

\section{Implementation}

The computer program was written in Fortran, and all simulations reported here were run on a Cray Y-MP/832 at the Pittsburgh Supercomputing Center. A single simulation of $40 \mathrm{sec}$ of neural time ( 80 stimuli $\times 500 \mathrm{msec} / \mathrm{stimulus)}$ for each of 100 neurons required $6.2 \mathrm{sec}$ of CPU timc. As described above, synaptic weights and connections among the neurons were determined probabilistically, with only means and ranges being prespecified. Therefore, the detailed structure of the model varied from one simulation to the next, even though the same values were used to establish the anatomy. Different simulations using identical thalamic input files and identical anatomical parameter values thus produced a range of results. Therefore, each simulation presented here was repeated 6-10 times, and the means \pm 1 standard deviation of output variables were computed.

\section{Data analyses}

Each stimulus presentation produced a spike train for each model neuron. These data were summed over all angles of stimuli on a msec-bymsec basis to produce for each cell a peristimulus time histogram (PSTH). From these, numerical measures of cell activity during different time periods were calculated separately for spiny and smooth cells. These 


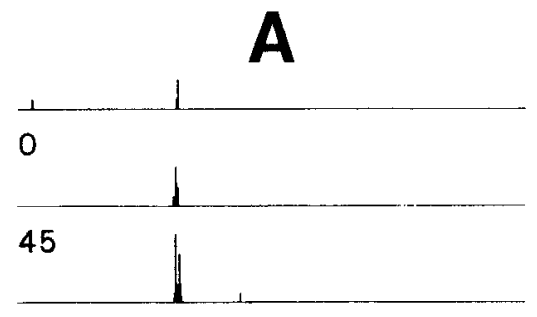

90
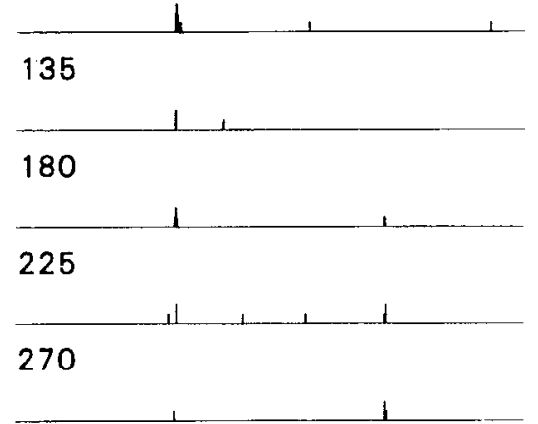

315

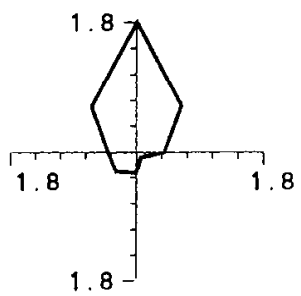

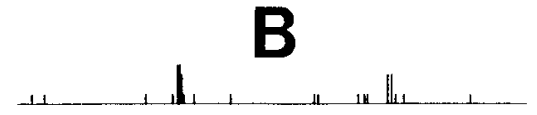
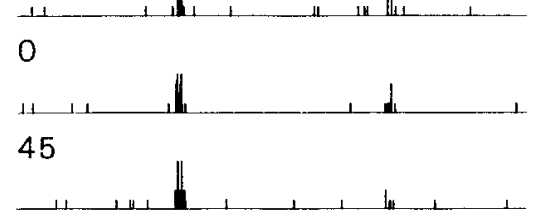

90

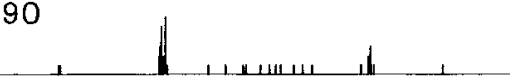

135

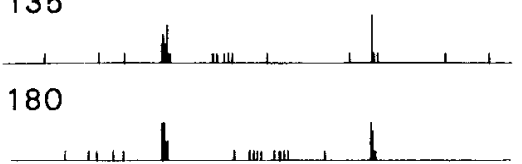

225

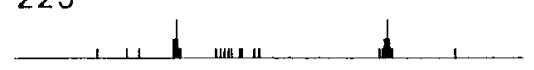

270
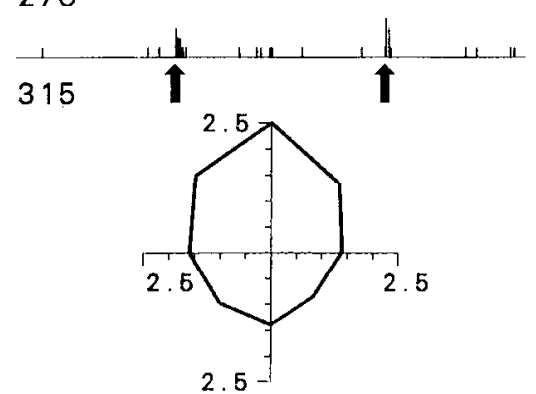

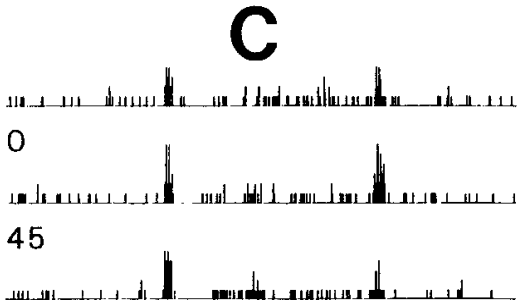

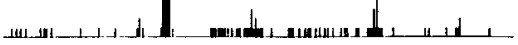

90

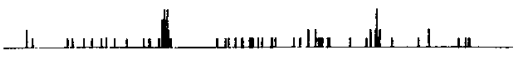
135

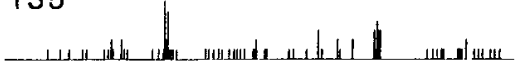

180

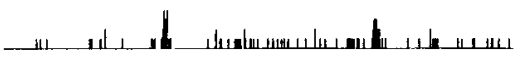

225

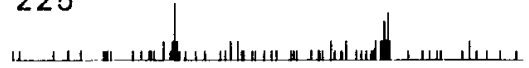

270

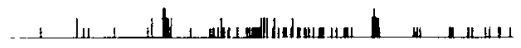

315

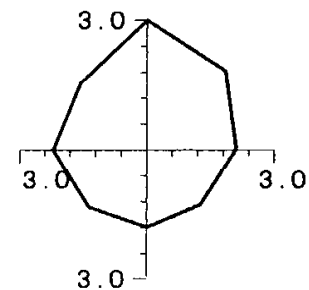

Figure 2. Responses to different angles of whisker deflection of three representative model neurons. $A$ and $B$ show data from spiny cells; $C$, from a smooth cell. PSTHs show accumulated responses of each neuron to 10 deflections at eight different angles. $0^{\circ}$ represents an initially caudal deflection; $90^{\circ}$, an upward deflection. Arrows in $B$ denote stimulus onset and offset. Duration of the PSTH is 500 msec, and individual tick marks represent bins of 1 spike/msec. Polar plots show ON responses in average spikes/stimulus in polar coordinates, where caudal is to the right. All three cells display an upward directional selectivity because each thalamic input file was "rotated" to have its maximal response at the upward deflection (see Materials and Methods). Note the broader tuning and greater spontaneous and stimulus-evoked activities of the smooth cell.

measures took the form of average numbers of spikes per stimulus. ON and OFF responses were measured for periods of $20 \mathrm{msec}$ following stimulus onset and offset, spontaneous (prestimulus) activity for 100 msec, and plateau activity for $125 \mathrm{msec}$ beginning $50 \mathrm{msec}$ after stimulus onset.

Spike counts from real and simulated neuron populations were compared separately for RSU/spiny cells and FSU/smooth cells. Output parameters included the magnitudes of spontaneous activity and of $O N$, plateau, and OFF responses for all eight deflection angles combined. Comparisons also were made between simulated and real responses for the pattern of activity evoked in RSU/spiny cells during the stimulus plateau; measured parameters included (1) the latency of the activity peaks, (2) the magnitude of the peaks relative to the troughs, and (3) the veridicality of the overall pattern ascertained using a waveform matching algorithm.

A single descriptor, hereafter referred to as SCORE13, was derived whose value depended multiplicatively on nine aspects of the output: smooth cell $O N$ and plateau responses, spiny cell spontaneous activity, latency of OFF response, plateau peak-to-trough ratio, ON response magnitude, plateau response magnitude, plateau waveform matching, and $\mathrm{ON}$-to-OFF ratio, derived by dividing $\mathrm{ON}$ response magnitude by the size of the OFF response. The latter four measures were deemed particularly important because they represented a broad spectrum of stimulus-evoked activities, including magnitudes of strong and weak response components and their temporal patterns. To emphasize thcir importance in the analyses, their values were squared. Each of the nine output measures was incorporated into a factor, $F$, of the following form:

$$
F=\max \{1-(|S-R| / \alpha R), 0\}
$$

where $S$ is the obtained simulated value, $R$ is the desired value from real barrel neurons, $\alpha$ is a stringency factor, and the maximum value notation indicates that $F$ was set to 0 if the function had a negative value. Normally $\alpha$ was $\mathrm{I}$, and $k$ varied from 0.0 to 1.0 to 0.0 as $S$ went from 0 to $R$ to $2 R$. For two measures (spiny cell spontancous activity and OFF response latency) we made $F$ less stringent by setting $\alpha=2$. SCORE13 was the product of these factors, so that a value of 0.0 for any single factor yielded a 0.0 for the whole function. Thus, SCORE13 was a stringent descriptor of the overall accuracy of a simulation, identifying areas of parameter space that reproduce all aspects of the output at least moderately well.

For cach simulation, an output file was generated that contained its parameter values and the obtained output. For off-line treatment, data files were transferred via Ethernet to an ALR FlexCache 33/386Z, and were analyzed and plotted using a data base management and statistics package (SPSSPC+, SPSS, Inc.) and graphics software (PLOTIT, Scientific Programming Enterprises, Inc). More than 35,000 different simulations were run, many of which explored the use of parameters and simulation techniques not described here. For characterizing the present model, we ran approximately 5000 simulations using nonidentical parameter values.

\section{Results}

Responses of individual neurons. All results, unless otherwise specified, are based on the set of parameter values that produced the best overall output (see Table 1). Although our analyses focused on population responses, we did examine PSTHs from individual cells to ascertain whether they were at least qualitatively similar to those obtained from real neurons. Figure 2 shows representative data from two spiny cells and one smooth cell obtained from a single simulation using the standard pro- 
REAL

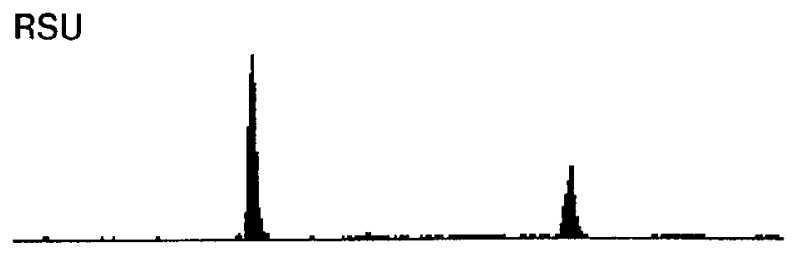

SIMULATED

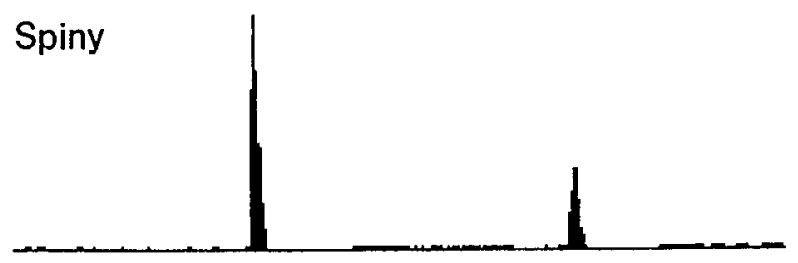

TCU

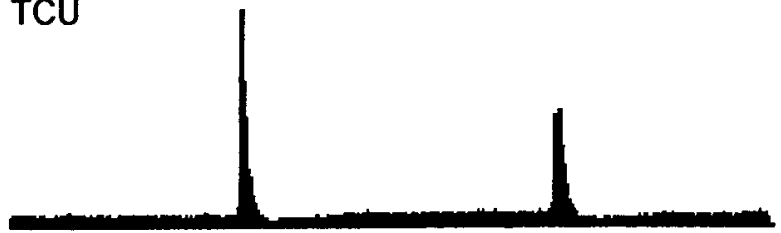

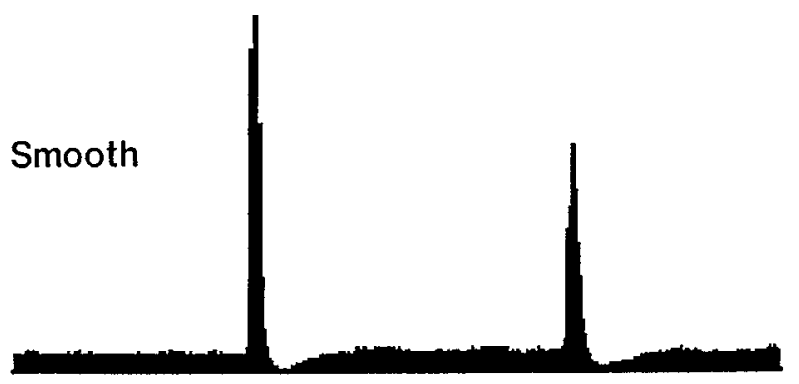

VB

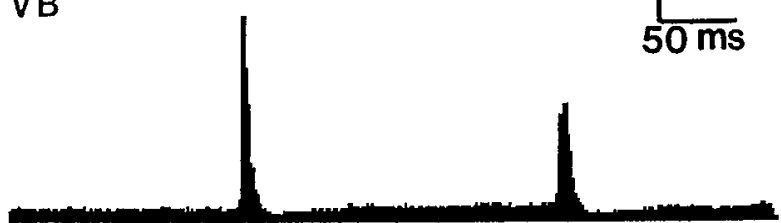

Figure 3. Population profiles from standard whisker deflection protocol. Each PSTH shows accumulated responses from all cells to 10 repetitions of all eight angles of PW deflection. Data on the left are from 242 RSUs, 16 FSUs, and 135 TCUs; data on the right, which are based on 10 simulations, are from 700 spiny and 300 smooth cells that were activated by 100 (VB) of the 135 TCUs. Vertical scale shows the probability that an action potential occurred in a $1 \mathrm{msec}$ bin. Note that this scale applies to all PSTHs.

tocol. Each panel consists of PSTHs based on 10 deflections of the PW at each of eight stimulus angles and a polar plot depicting the magnitudes of the $\mathrm{ON}$ responses. The spiny cell depicted in Figure $2 A$ was not spontaneously active and responded primarily to stimulus onsets. By contrast, the spiny cell of Figure $2 B$ showed some spontaneous activity and responded vigorously to both stimulus onset and offset. Note that this cell's plateau response varies with stimulus angle. The responses of these simulated spiny cells are typical of those observed in real barrel RSUs, and both real RSU and simulated spiny ccll populations display unimodal distributions of response magnitudes (data not shown). The smooth cell of Figure $2 C$ displays the characteristic high spontaneous and stimulus-evoked activity of real FSUs. As in the real barrel cell populations, simulated smooth cells respond vigorously to more angles of whisker deflection than do spiny cells. Using rotated input files, as done here, the selectivity that simulated neurons show for whisker deflections at a particular angle, in this case upward or $90^{\circ}$, is exaggerated relative to real barrel neurons. For the remainder of the Results, findings are reported for data combined over all eight angles.

Population responses. Figure 3 compares population PSTHs from real and simulated barrel neurons. Each response profile was constructed by a bin-by-bin summation of all spike trains obtained with the standard protocol. Differential responsiveness is the overriding characteristic that distinguishes these three cell types. The FSU/smooth cells are twice as active as TCUs with respect both to spontaneous and stimulus-evoked activity, whereas RSU/spiny cells have sevenfold less spontaneous activity than the TCUs despite having nearly equally sized ON responses. Thus, the RSU/spiny cells, unlike the FSU/smooth cells, show a large increase in signal-to-noise ratio. The only noticeable difference between the real RSU and simulated spiny cell profiles is that $\mathrm{ON}$ and $\mathrm{OFF}$ peaks for the latter are somewhat taller and narrower, which is due at least in part to the instantancous rise times of the modeled PSPs. Profiles for FSUs and simulated smooth cells are discussed below.

Attention to low-level activities provided further opportunities to constrain model parameters. We focused on reproducing the average levels of spontaneous activity and plateau responses, as well as the pattern of plateau activity in the RSU population. Figure 4 shows the same histograms as Figure 3, but magnified to reveal details of the activity. As in real RSUs, simulated spiny cell activity decreases immediately after the ON and OFF responses. Such a decrease occurs also in the VB response, but this does not account entirely for the postexcitatory suppression observed in the model. The time course of the latter depends on the IPSP decay constant, and the depth of the postexcitatory trough is considerably less when all smooth cell outgoing synaptic weights are set to 0 . Moreover, postexcitatory response suppression is obtained in the model barrel even when 
REAL
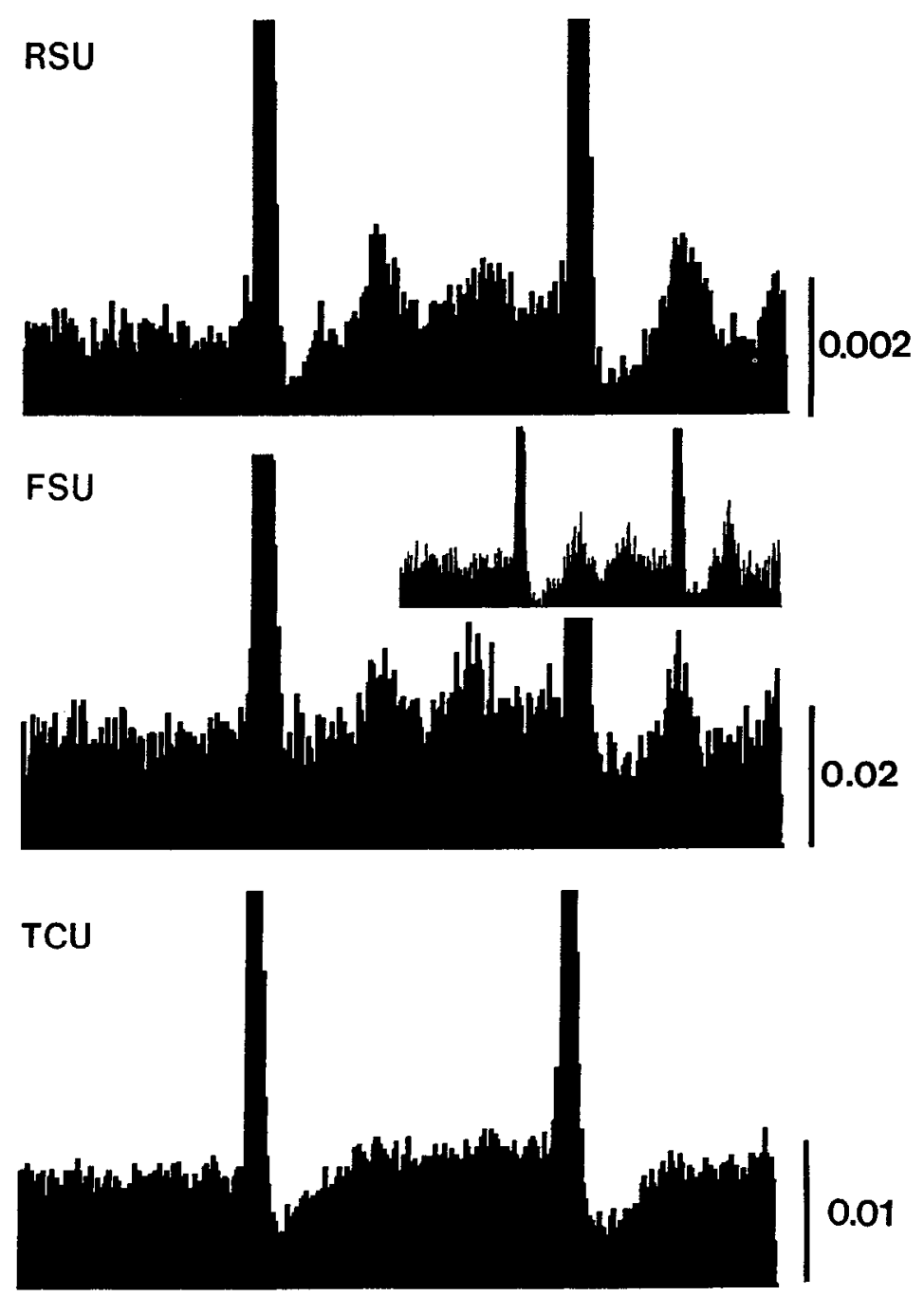

SIMULATED
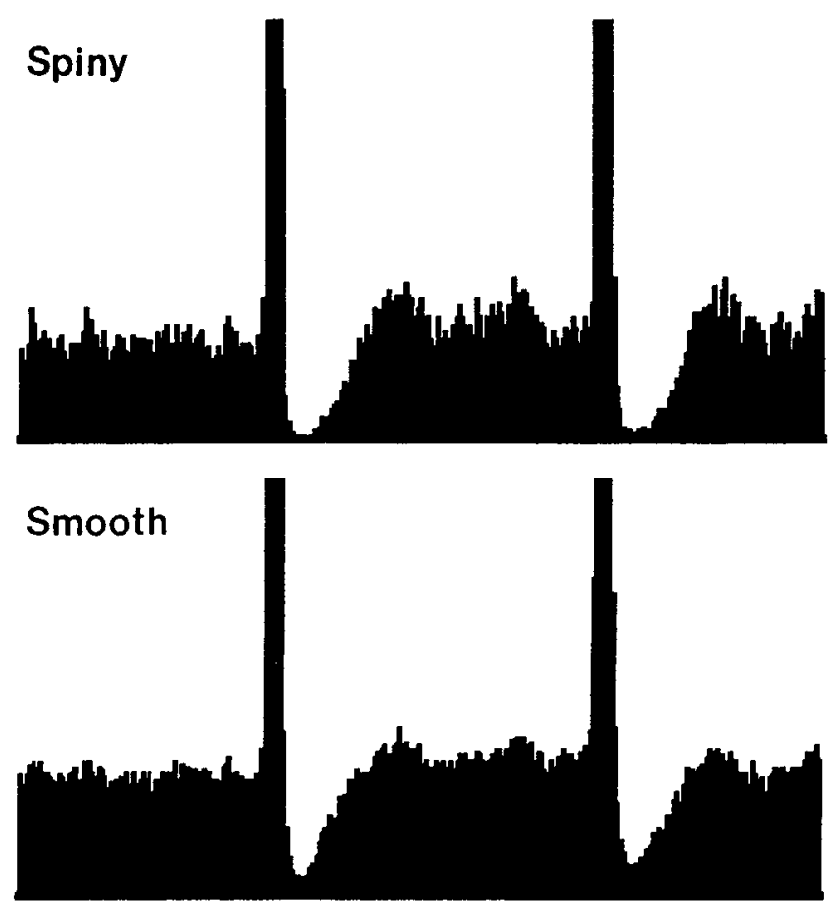

VB

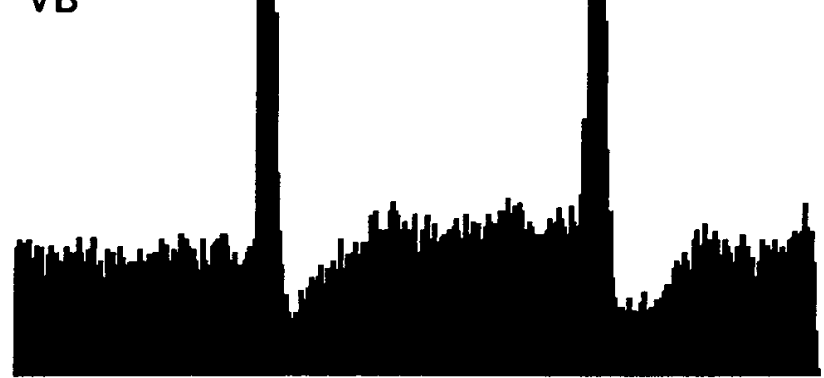

Figure 4. Expanded population profiles from Figure 3. The vertical axes have been magnified to illustrate low level activities. For each cell type, real and simulated data are plotted with the same scale. All PSTHs have been scaled so that the height of the spontaneous activity bins are comparable; note that the spontaneous activity of FSU/smooth cells is approximately 15 times that of RSU/spiny cells and two times that of thalamic cells. The inset in the FSU panel shows responses of the FSU subpopulation (12 of the 16) that displayed postexcitatory response suppression. The inset is reduced 50\% relative to the large FSU PSTH.

it is absent in the input files, ascertained by using, as input, data from slowly adapting trigeminal ganglion cells (see Fig. 2 in Lichtenstein et al., 1990).

For spiny cells the model network also reproduces reasonably well the size and timing of secondary peaks and troughs following the initial postexcitatory suppression. As with the real data, similar activity follows the simulated OFF response. These patterns are enhanced relative to the VB response. As is the case for postexcitatory response suppression, inhibition is required to produce them accurately. With inhibitory synaptic weights of 0 , only a very subtle cyclic pattern can be obtained, even when other parameters are adjusted to achieve the best results (data not shown).

The population response of FSUs is more complicated. Simons and Carvell (1989) reported that 4 of their 16 observed FSUs showed no poststimulus response suppression (see their Fig. $15 C$ ). The inset in the real FSU population profile in Figure 4 shows the behavior of the 12 FSUs that display postexcitatory suppression. Comparison with the right panel shows that the pronounced decrease in the activity of the simulated smooth cclls following the ON response is more similar to these 12 FSUs than to the overall FSU population. We did not explicitly model FSU subpopulations. If postexcitatory response suppression in real barrel cells depends on activity in a subpopulation of FSUs that continue to fire during this period, then the model compensated for the absence of this group by having relatively longer lasting IPSPs, whereby the modeled smooth cells' large ON and OFF responses produced strong postexcitatory inhibition.

$O N$-to-OFF ratio. An important difference among the responses of the three cell populations illustrated in Figure 3 is the magnitude of their OFF responses. In particular, RSU OFF responses are much smaller than those of TCUs even though their ON responses are approximately equal. To quantify these relationships, an ON-to-OFF ratio was computed by dividing the mean ON response by the mean OFF response. Data from 


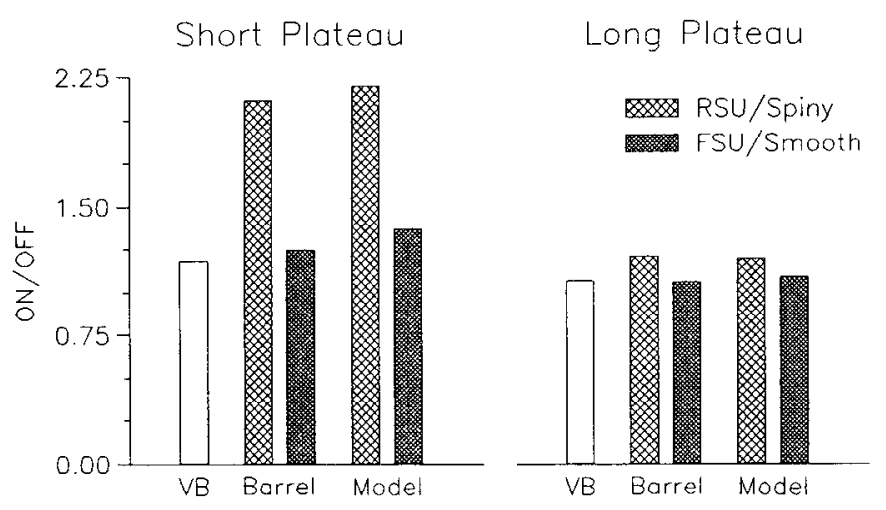

Figure 5. ON-to-OFF ratios. The left panel shows data for the standard protocol in which a 200 -msec-long stimulus plateau was used; for the right panel, the stimulus lasted $1400 \mathrm{msec}$. With the long stimulus, the relative size of the OFF response increases disproportionately in the RSU/spiny population. Note the similarities in both panels between ON-to-OFF ratios of VB and FSU/smooth cells.

the thalamic input cells and from the real and simulated barrel cells are shown in the left panel of Figure 5. The model network reproduces the large spiny cell ON-to-OFF ratio and the somewhat smaller ratio for the smooth cells.

The accurate reproduction of the $\mathrm{ON}$-to-OFF ratio is critically dependent on temporal integration factors. Close inspection of the input data files reveals that the OFF responses of thalamic neurons are more temporally dispersed than their $\mathrm{ON}$ responses; on average input spikes to a barrel cell from the same or different thalamic neurons occur at longer intervals. One way of exploiting this difference in input timing to produce the desired $\mathrm{ON}$ to-OFF ratio was to use a relatively rapid EPSP dccay, and this was the strategy we adopted. By limiting temporal summation, an EPSP decay time constant of 3-5 msec emphasized differences in the timing of the input spike trains. EPSPs with yet more rapid decay (time constants of 1 or $2 \mathrm{msec}$ ) have the effectiveness of their temporal summation so reduced that realistic output for many response variables could not be obtained. By altering other temporal factors, appropriate ON-to-OFF ratios can be obtained with much longer EPSP decay constants, for example, a value of $17 \mathrm{msec}$ as reported recently for pyramidal cells in rat visual cortex (Mason et al., 1991). One strategy is to employ a slower, that is, noninstantaneous, IPSP rise time; such IPSPs disproportionately suppress slowly developing responses, such as those to stimulus offset. Another approach is to have thalamic inputs to spiny cells sum nonlinearly, such that a disproportionately large response results from more synchronous inputs, that is, during the ON response (sec Shepherd et al., 1985).

A second temporal factor that greatly affects the ON-to-OFF ratio is the refractory period. Refractoriness constrains the size of responses since it limits the frequency of burst discharge under highly depolarized conditions. The dampening effect of a refractory period is greater for the stronger and more rapidly developing $\mathrm{ON}$ response than for the less vigorous and more ternporally dispersed response to stimulus offset. In effect, it prevents ON-to-OFF ratios from becoming too large. Therefore, as modeled here, a proper ON-to-OFF ratio reflects a balance between two opposing factors: EPSP decay constants that exaggerate differences between $O N$ and $O F F$, and refractory periods that limit them. Additionally, as will be discussed below, ON-to-OFF ratios are influenced by the relative strengths of thalamic and intrabarrel synapses.
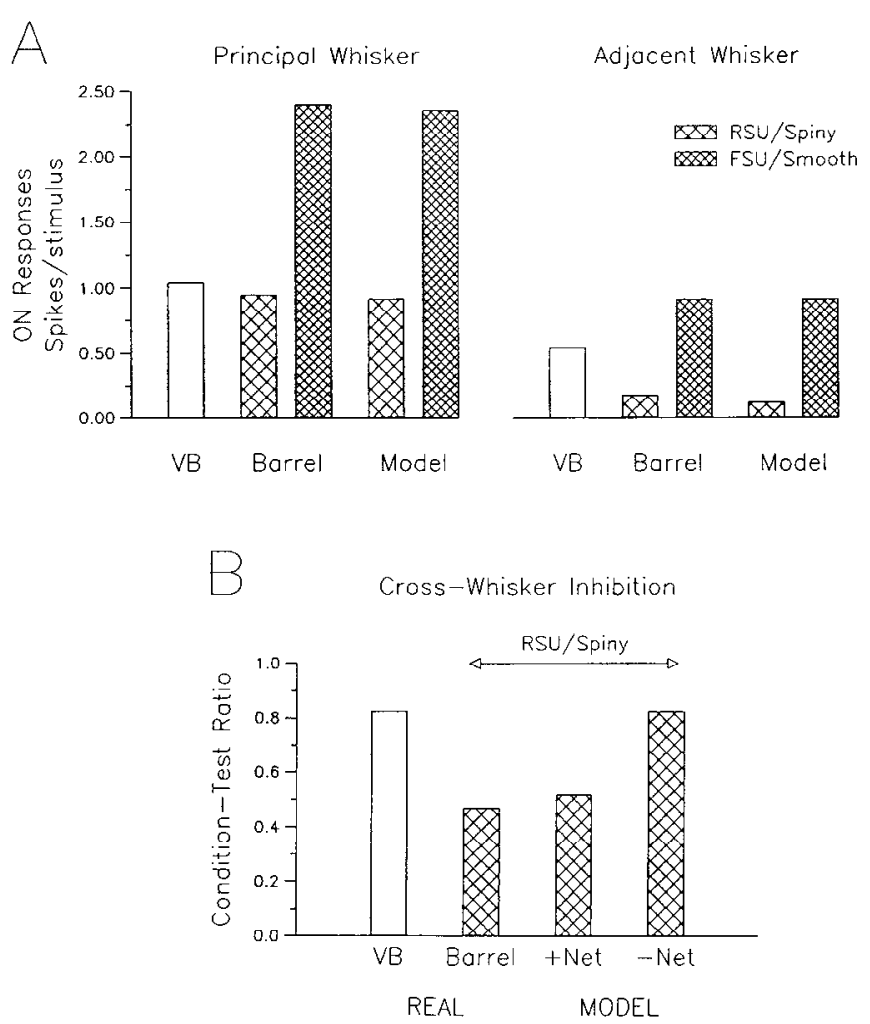

Figure 6. Receptive field organization. Excitatory components of the receptive fields are represented in $A$, which shows $O N$ response data obtained with the standard protocol (left, PW) and the multiwhisker deflection protocol (right, adjacent whisker). Compared to VB, note the disproportionately small RSU/spiny cell adjacent whisker response and the relatively large FSU/smooth cell adjacent whisker response. $B$ quantifies surround inhibition observed in VB and RSU/spiny cells. To compute the condition-test ratio, the $\mathrm{ON}$ response of the $\mathrm{PW}$ when it was deflected $20 \mathrm{msec}$ later than an adjacent whisker was divided by the PW response when it was deflected alone. Simulations were run with $(+N e t)$ and without $(-N e t)$ a network, that is, in the presence and absence of synaptic interconnections among barrel neurons. Without a network, the normal level of cross-whisker inhibition cannot be produced (see text).

Compared to spiny cells, smooth cells, which receive stronger thalamic synapses and which are inherently more excitable, are less dependent on temporal summation. Therefore, their responses are more reflective of thalamic input activity, with their ON-to-OFF ratios being smaller than those of spiny cell/RSUs.

Long plateau protocol. After a set of parameter values had becn identificd that accurately reproduced a wide variety of output characteristics for the standard protocol, we tested the ability of the same model to simulate appropriate responses to other whisker stimuli. One set of experiments used whisker deflections lasting $1400 \mathrm{msec}$. Data from real and simulated barrel neurons are summarized in the right panel of Figure 5. $\mathrm{ON}$ responses were unaffected by the increased stimulus duration. The VB OFF response for the long plateau protocol was slightly larger than for the short plateau, but more importantly, the spikes occurred within a shorter period of time. When these spike trains were used as input to the model network, spiny cell OFF responses were greatly increased for the reasons discussed above. This yielded an ON-to-OFF ratio much smaller than that obtained with the short plateau and one that was virtually identical to that of the RSUs studied using long-duration stimuli. 

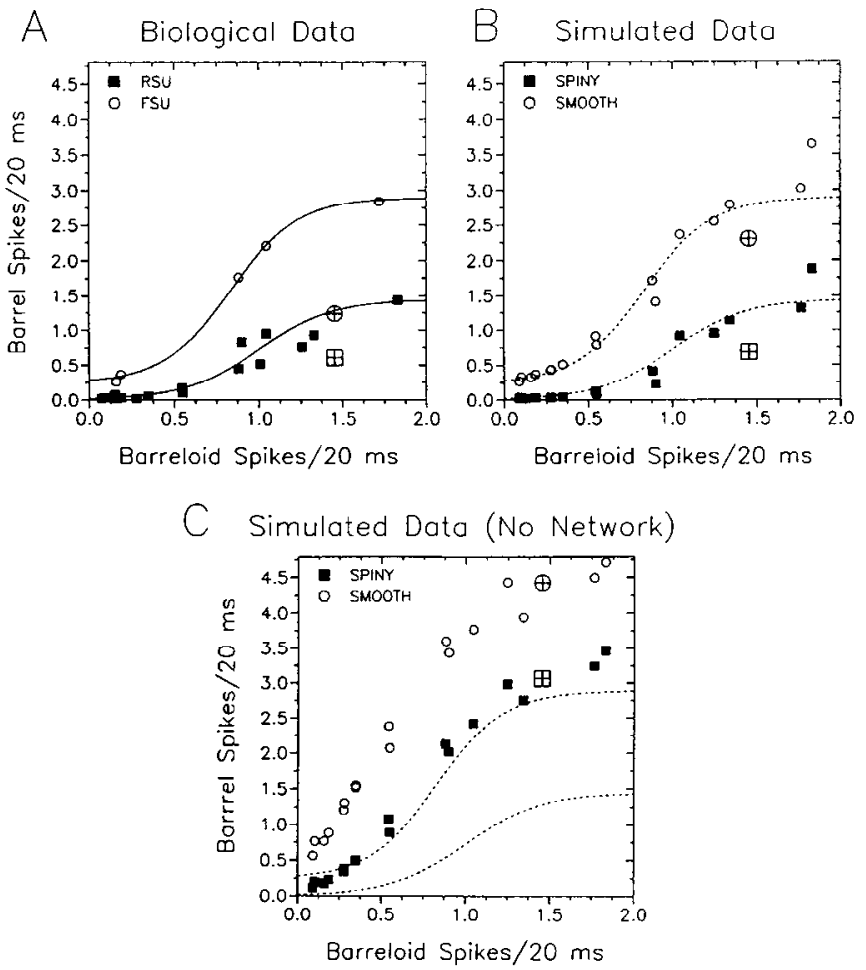

Figure 7. TC transfer functions. Scatterplots show corresponding activity levels for thalamic barreloid and cortical barrel cells. For the RSU data in $A$, the low values consist of spontaneous and plateau activities from the three stimulation protocols, and the progressively larger responses consist of adjacent whisker OFF, adjacent whisker ON, PW OFF for short plateau, PW OFF for long plateau, PW ON, and PW maximal angle ON. Because of small sample sizes, FSU data are from the standard protocol only. For the simulated data in $B$ and $C$, all responses are plotted. Curves, which were fitted (see text) to the real data in $A$, were transposed without alteration to the other panels. Large crossed symbols represent conditioned-test $\mathrm{ON}$ responses obtained with the multiwhisker protocol.

Multiwhisker deflection protocol. The model was also tested for its ability to simulate spatial features of barrel neuron receptive fields. For these experiments, the PW and one of its immediately adjacent neighbors were deffected $20 \mathrm{msec}$ apart in a condition-test paradigm wherein the adjacent whisker was deflected first (see Materials and Methods). The left panel of Figure $6 A$ quantifies PW ON response data for the population profiles shown in Figure 3 above. Adjacent whisker ON responses are summarized at the right. On average the response of TCUs to adjaccnt whisker deflections is approximately half the size of their response to deflections of the PW. For the simulated spiny cells, the adjacent whisker response is about one-eighth (0.13) of the PW response, despite the fact that their PW responses are only slightly smaller than those of thalamic neurons. Similarly, real RSUs display a disproportionately small adjacent whisker response $(0.19 \times \mathrm{PW})$.

Because of the small sample size of FSUs obtained with the multiwhisker protocol, detailed comparisons between real and simulated data are unwarranted. An important finding, however, is that the simulated smooth cell response to adjacent whisker deflection is considerably more vigorous than that of the spiny cells. Figure $6 B$ shows that the former produces crosswhisker inhibition in the model barrel. Simulations were run with and without a network, that is, in the presence or absence of connections among barrel cells. With the network intact, the conditioned-test response was reduced by $48 \%$ in spiny cells, compared to a $52 \%$ reduction in real RSUs. By contrast, paired whisker deflections result in only an $18 \%$ reduction in the conditioned-test response observed in the thalamus. Importantly, when all intrabarrel synaptic weights are set to zero, the condition-test ratio of spiny cells is virtually identical to that of the VB cells.

In the absence of inhibition provided by smooth cells, stimulus-evoked activity of all barrel neurons increases to levels well above the physiological range (see Fig. $7 \mathrm{C}$ ). Before conducting the condition-test experiments without network connections, we therefore reduced TC synaptic weights and adjusted firing threshold functions to achieve appropriate levels of network excitability, determined by spontaneous activities (low-level excitation) and ON responses (high-level excitation) for the standard protocol. Since even such optimized networks failed to show response suppression, we conclude that the large reduction in the conditioned PW response of spiny cells requires active inhibitory processes within the model barrel. Also, under these conditions the adjacent whisker $\mathrm{ON}$ response increased by $36 \%$ even though the PW ON response was unchanged. In other words, the difference between the PW and adjacent whisker responses was less pronounced than with the network intact. Thus, for reasons discussed below, network interconnections contribute to the disproportionate enhancement of the larger (PW) response relative to the weaker (adjacent) one.

Thalamocortical transfer functions. Quantitative comparisons of real and simulated barrel data are summarized in the scatterplots of Figure 7. In each panel, data points show the magnitudes of corresponding responses obtained from thalamic barreloid and cortical barrel neuron populations. For constructing the curves through the data points we used Equation 3, the model's activation function, and empirically adjusted its parameters to achieve a good fit to the real data, which are shown in Figure $7 A$. These curves were transposed without alteration to Figure $7 B$, where the points represent simulated data. For constructing the curves, conditioned-test responses (larger, crossed symbols) were excluded; they were unique in that they followed deflections of another whisker. The curves derived for the biological data describe the simulated data remarkably well. Taken together, the scatterplots demonstrate that the transformations that occur within a barrel are nonlinear and that FSU/ smooth cells are uniformly more active than RSU/spiny cells.

The points representing the conditioned-test response of both real and simulated spiny cells fall considerably below the fitted curves. As in Figure $6 B$, these findings underscore the effectiveness of adjacent whisker deflections in engaging inhibitory circuitry within the network. When all network connections are removed, the transfer functions shown in Figure $7 \mathrm{C}$ are obtained. Responses of both spiny and smooth cells are greatly increased, and conditioned-test responses lie on the same line as the other points. These findings indicate that, despite the numerical predominance of spiny cells and their excitatory synapses (see Table 1), the overall effect of the network is inhibition. This reflects the fact that smooth cell spontaneous activity normally is 15-fold greater than that of spiny cells and that the modeled IPSPs are larger and last longer than the EPSPs (see also Discussion).

Tension. Figure 8 shows results from a series of experiments designed to investigate the extent to which good output could be obtained using different balances of thalamic drive, network drive, and network inhibition. We define a term, called tension, 

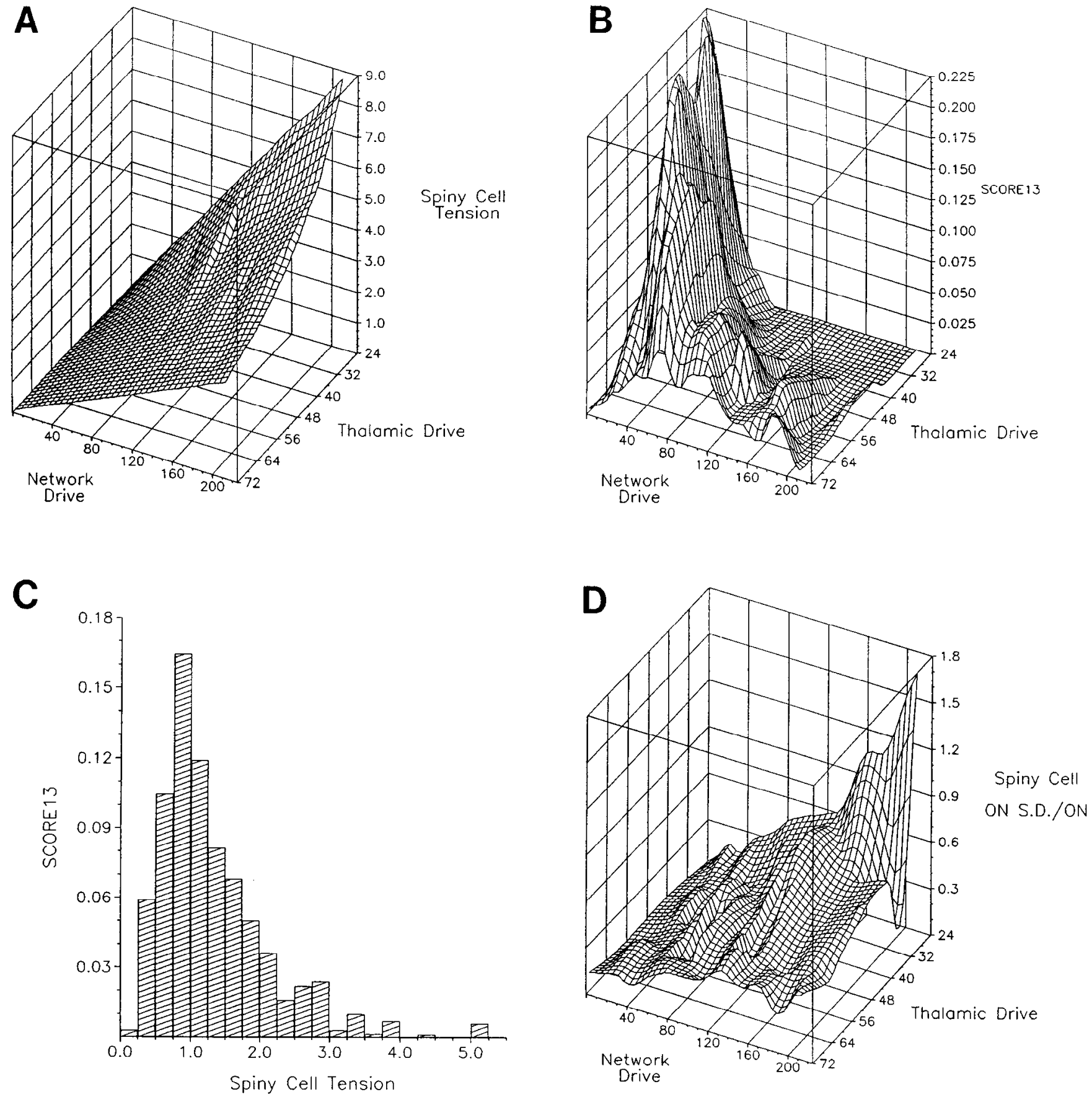

Figure 8. Tension. Different values of thalamic and network drive were obtained by varying TC and Ex synaptic weights, keeping synapse numbers constant. Increases in drive were matched by approximately proportional increases in inhibitory weights, but the latter axis was not plotted due to dimensional limitations. $A$ shows how tension, defined as network drive divided by thalamic drive, varied in the experiments whose results are illustrated in the other panels. Note that both drive levels are largest at the nearest edge of the box. $B$ shows how a composite measure of output, $S C O R E 13$, varied as a function of network and thalamic drive. Binned data are plotted in $C$ as a function of spiny cell tension. The ridge in $B$ follows a line of relatively constant tension (compare with $A$ ), which is shown in $C$ to represent a condition in which network drive and thalamic drive are approximately equal. $D$ demonstrates that as tension increases (compare with $A$ ) so does output variability, shown here using average ON responses and their SDs. For all panels, results are based on 74 data points, each representing data from six simulations.

to be the ratio of intrinsic to extrinsic excitatory synaptic drive impinging on a given cell population, where drive is the product of average synaptic weight and average number of synapses. Figure $8 A$ shows how tension varied in these experiments. Thalamic drive is plotted as the product of the average TC synaptic weight onto spiny cells and the average number of TC synapses onto spiny cells; thalamic drive onto smooth cells was adjusted proportionally (values not shown). Network drive is the product of the average spiny cell synaptic weight and average spiny cell convergence onto barrel cells. For each intrinsic/extrinsic drive combination, inhibition within the network was varied by adjusting smooth cell synaptic weights to maintain spiny and smooth cell ON responses within their proper ranges $( \pm 10 \%)$. Importantly, increases in either thalamic or network drive always required approximately linear increases in inhibitory synaptic weights. Seventy-four drive combinations were examined, 
and for each combination six simulations using the standard protocol were run and the results averaged. The three-dimensional surfaces were interpolated from these 74 points. No other parameters were altered. Due to dimensional limitations, the inhibitory parameter axis was not plotted.

In Figure $8, B$ and $C$, the dependent variable is SCORE13, a composite measure of the overall accuracy of a simulation's output (scc Matcrials and Methods). The ridge of the threedimensional surface in Figure $8 B$ describes a curvilinear relationship between intrinsic and extrinsic drive. Beginning at high drive levels (nearest edge of box), the ridge curves gradually and rises as it moves in the direction of decreasing network drive, curves and rises rapidly at intermediate levels of thalamic and network drive, and gradually again as it moves in the direction of decreasing thalamic drive. Along the network drive axis, the ridge is relatively broad at high levels of thalamic drive and narrows progressively as thalamic drive decreases. The data illustrate three principal findings. First, good simulation results can be obtained over fairly broad ranges of thalamic or network drive. Second, as thalamic drive increases, and as inhibition is increased accordingly, intrinsic drive also must increase to produce good output. Indeed, tension is relatively constant along the ridge (see below). Third, when the input drive is weak, the network must be more precisely "tuned" than when it is strong, evidenced by the pronounced narrowing of the ridge as thalamic drive decreases.

The importance of maintaining a proper balance between intrinsic and extrinsic drive is further illustrated in Figure $8 C$, where SCORE13 values are plotted as a function of spiny cell tension. Note that the best simulation results were obtained when intrinsic and extrinsic drives were approximately equal. Poor output was obtained at low tension levels for reasons discussed below. One reason for poor output at very high tension levels was a large variation in output from one simulation to the next. To characterize this variability, we computed the means and intersimulation SDs of the spiny cell ON responses obtained at each data point and divided the SD by the mean. ON responses were analyzed because simulation parameters were always adjusted to maintain the average ON response at $\pm 10 \%$ of its correct level. Comparison of the obtained results, shown in Figure $8 D$, with the data of Figure $8 A$ illustrates the approximately linear relationship between variability and tension. We conclude that the greater the tension, the more chaotic the network's behavior, being more influenced by its own activity and less influenced by its external (thalamic) inputs.

These results indicate a need for some network drive in the model. In order to determine whether excitatory connections within the network were absolutely necessary for the model to the produce realistic output, we removed them, reducing tension to 0 . Total thalamic drive to both spiny and smooth cells was increased to compensate for the loss of the intrinsic network excitation, and parameters were adjusted to produce spontaneous activities and $\mathrm{ON}$ responses in their physiological range. Under these conditions, spiny cell OFF responses were consistently too large. Careful examination of the response profiles revealed that their OFF responses rose more rapidly than in the "normal" network. There are two major reasons for this decreased ON-to-OFF ratio, both of which derive from the positive feedback nature of recurrent excitation. First, recurrent excitation is positive feedback and, as such, produces highly nonlinear voltage changes. Eliminating such feedback necessarily lessens the voltage differential, and hence the response dif- ferential, between large and small inputs. Since ON responses were maintained at the proper level, the OFF responses became larger. Second, recurrent excitation helps produce responses that are more temporally dispersed and hence, as described above, more subject to the effect of the relatively short EPSP duration. Without such excitatory feedback, and with stronger TC drive, the spiny cell OFF response, much more so than its already fairly synchronous $\mathrm{ON}$ response, has its shape altered to resemble more closely that of its input. Consequently, a greater proportion of their spikes now occur before the disynaptic feedforward inhibition in the network temporally summates to exert its strongest effect. Conversely, when spiny cell tension is increased, the OFF response becomes too small relative to $O N$. This reflects excessive positive feedback within the network that increases the nonlinearity of the voltage responses and causes further temporal dispersion of the OFF response. Together, these effect too great a separation between large (ON) and small (OFF) responses along the sigmoid activation curve.

Convergence and grain. Good output could not be obtained when individual TC cells exerted excessive influence on cells postsynaptic to them. Such conditions occurred when the average TC convergence onto barrel cells was lowered and the thalamic synaptic weight was correspondingly increased to maintain appropriate $\mathrm{ON}$ response levels. In particular, with no convergence, that is, when each barrel cell received thalamic input from only one neuron, the requisite TC synaptic weight was very high and overall good output could not be obtained under any conditions. We consider low convergence networks to be too "grainy" in that barrel cell action potentials are too readily evoked by activity in only one or a few thalamic axons.

A series of simulations were performed in which TC convergence was varied and mean TC synaptic weight was adjusted to keep the product of the two, and hence the thalamic drive, constant. We examined thalamic drives corresponding to levels $36.0,48.0$, and 72.0 in Figure $8 B$; intrinsic drive was varied as in the tension experiments and inhibitory synaptic weights were set accordingly. At the three thalamic drives, minimum averages of $4.5,6.0$, and 9.0 thalamic inputs per spiny cell, respectively, were required to obtain SCORE1 3 values of $>0.0$. Each of these convergences was associated with a grain of 0.5 , where grain is a normalized synaptic weight, defined as the average synaptic weight divided by the difference between the threshold and the resting potential. For a grain of 0.5 , a single thalamic synaptic event could depolarize a spiny cell from rest halfway to its firing threshold. Similarly, for spiny cell divergent connections, which have the same EPSP decay time constants, a grain of $>0.5$ was also associated with poor output. For the model's smooth cell divergent connections, which have IPSP decay constants three times those of the EPSPs, a grain size greater than about 0.17 was associated with poor output. If one defines synaptic impact as the product of weight grain and PSP decay constant (units of $\mathrm{msec}$ ), then for all three synaptic populations, a synaptic impact less than $\sim 2.5 \mathrm{msec}$ was required to achieve even marginally acceptable output, and values of $\sim 1.5 \mathrm{msec}$ were necessary for optimal output. Taken together, the results indicate that some minimum degree of primary (TC) and secondary (Ex and In) convergence is necessary for the faithful reproduction of the model's response. Stated differently, neurons require a reasonably broad sampling of their input populations, accomplished here by TC convergence and network interconnections.

For each of the three thalamic drive levels examined in detail, increasing the TC convergence improved output, to a certain 
point. Figure $9 A$ shows results obtained with a thalamic drive of 72 ; at the convergence value of 12 , simulation parameters were identical to those used for the "slice" in Figure $8 B$ where thalamic drive equals 72.0. Note the improvement in composite output that is obtained as a given spiny cell receives thalamic input from a larger number of thalamic cells, to a maximum of approximately 18.0. Examination of individual output parameters indicated that graininess has a particularly strong effect on those aspects of the network's spike activities that are weakest. Figure $9 B$ shows the effect of TC convergence on the transformation of spiny cell spontaneous activity and ON and OFF responses. As convergence decreases, the characteristically small spontaneous activity and OFF response become larger, whereas the ON response is essentially unchanged. When a spiny cell receives low input convergence and correspondingly large TC weights, the roughly sevenfold higher spontaneous activity present in the few thalamic neurons that contact it is more directly reflected in the spiny cell's output. Similarly, the paucity of spatial integration yields spiny cell OFF responses that too closely mimic the large thalamic OFF response. Conversely, with high convergence the weight of any given thalamic synapse is so small that spiny cell action potentials are evoked only in the unlikely circumstance of spikes occurring on many thalamic inputs at more or less the same time. This explains the overly small spontaneous and OFF response levels at the highest convergences. ON responses are less affected by high convergence because a large proportion of the thalamic cells are activated by stimulus onset. Finally, the dampening effect of the very fine grain associated with high convergence can be partially, but not completely, negated by increasing the excitatory drive from the thalamus. Thus, in this serics of simulations, optimal output is associated with a limited range of grain size and TC convergence, a constraint that must exist, albeit to an unknown degree, in real barrels as well.

\section{Discussion}

The present study provided a quantitative evaluation of a number of factors that, together, may account for known differences between the response properties of TC projection neurons and those of neurons in layer IV of the rodent somatosensory cortex. The computational model was based on four major organizational principles: (1) nonlinear neuronal properties, (2) strongly responsive inhibitory and less responsive excitatory neurons, (3) monosynaptic, convergent inputs to both cell types from the thalamus, and (4) interconnections among and between neurons of both types. The model neurons themselves were physiologically unsophisticated, but the model network did incorporate neuron-like spatial and temporal integration factors, such as refractory periods, PSP decay constants, synaptic delays, and recurrent excitation and inhibition, and it was activated by real thalamic spike trains. Conceptually, the model occupies a middle ground between highly detailed, compartmental-type models of a single, or only a few, neurons, and highly abstract, massively parallel, connectionist-type neural network models. As such, it presently lacks the ability of the former to make precise estimates of specific physiological parameter values, but is more amenable to physiological interpretation than the latter. We found that the set of values that best reproduced barrel activity for one stimulus paradigm also enabled good simulations of the cortical response to other types of whisker stimuli. The model's functioning, though not simulated in full physio-
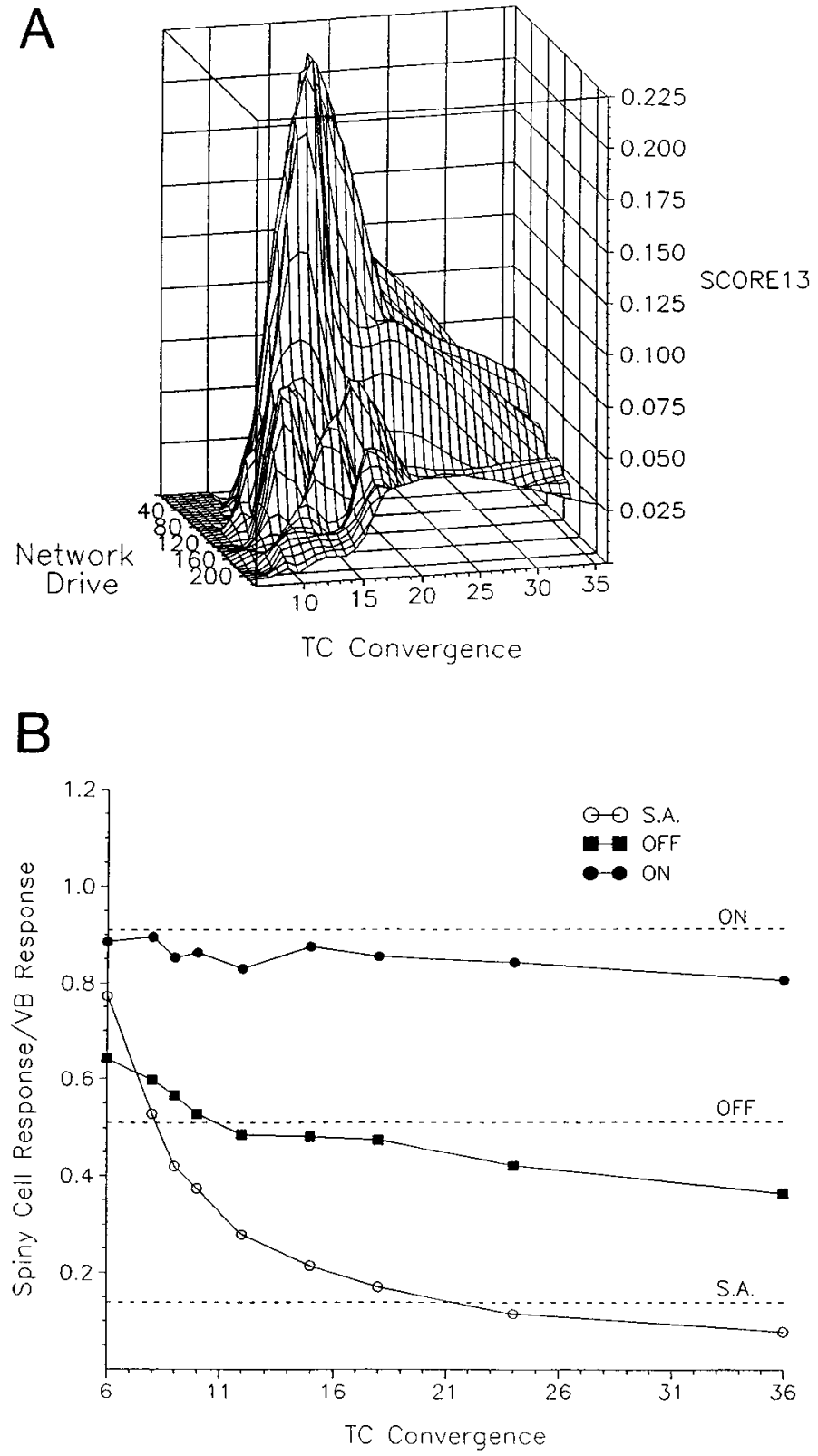

Figure 9. Convergence and grain. Network drive was varied in conjunction with $\mathrm{TC}$ weights and convergence, the latter of which is plotted. These were adjusted to maintain the level of thalamic drive constant at a value of 72.0. In $A$ the "slice" having a TC convergence of 12 is virtually identical to the "slice" of thalamic drive $=72.0$ in Figure $8 B$. $A$ shows that, to a maximum at 18 , increased convergence, and hence smaller grain, yields better output. $B$ shows that this improvement is due primarily to more accurate simulation of low-level activities. The ordinate is a measure of the change in the cortical response relative to its thalamic input. Curves show simulated data for varying amounts of TC convergence, as in $A$, and each point is the mean of values obtained at the different levels of network drive. Dashed lines indicate values for RSUs.

logical detail, may thus reflect important operational features of real barrels.

Nonlinearities of the model neurons. The neurons had two nonlinear characteristics: refractory periods and the sigmoid spike-generating functions. The former act disproportionately to reduce large versus small responses by limiting rapid bursts under highly depolarized conditions, whereas the latter do the opposite by approximating a step function that produces re- 
sponses selectively to the most depolarizing conditions. Although these functions are not implemented realistically in our model, both are features of real neurons, and both were critical in shaping the responsiveness of the model neurons.

The use of a voltage-based probabilistic activation function provided both deterministic and stochastic elements to spike generation. It helped prevent the model neurons from firing in lock-step synchrony during stimulus-evoked responses, and it permitted the model neurons to discharge occasionally even when their membrane potentials were substantially below our defined threshold. We found that the latter was necessary for generating appropriate levels of spontaneous activity within the network. The ability of neurons to fire by chance at low levels of depolarization had two effects. First, it imparted a small amount of background activity that was independent of thalamic activity. Second, it enabled the barrel neurons to be driven, albeit at low probabilities, by spontaneous activity in the thalamus. The need for these effects undoubtedly stems in part from the use of thalamic input files that had been obtained sequentially, in temporal isolation from each other. Such inputs necessarily lack synchrony in spontaneous discharges that may be present in vivo and that may be more effective in driving cortical "spontaneous" activity. Thalamic inputs evoked by whisker stimuli have, by their nature, more synchrony than does spontaneous activity and may therefore be more closely mimicked by our methodology.

Differential responsiveness of spiny and smooth cells. A second major operational feature of the model is that the network's inhibitory cells are more active than its excitatory cells. In real barrels, FSUs have high levels of spontaneous and stimulusevoked activities and smooth cells stain intensely for cytochrome oxidase (Land and Simons, 1985), and in behaving animals barrel cells that are most heavily labeled by 2-deoxyglucose are immunoreactive for glutamic acid decarboxylase (J. McCasland, personal communication). To achieve a differential responsiveness in modeled smooth and spiny cells, they were made to differ with respect to their intrinsic "biophysical" properties, the effectiveness of their TC synapses, and their synaptic relations with each other.

A number of simplifying assumptions are implicit in our modeling of the spiny and smooth cells. First, each model neuron was spatially dimensionless. The net effectiveness of proximal versus distal synapses was, however, explicitly modeled using synaptic weight. We also ignored potentially important spatial and/or temporal interactions among individual synapses distributed along a neuron's dendrite (Koch et al., 1983; Shepherd et al., 1985; Wilson, 1988). Possible nonlinearities associatcd with synapse location and/or timing may, however, have been captured by the slope of each model neuron's sigmoid activation function. A second simplification is that each cell class is treated as comprising a homogeneous population. Substantial variations in the morphologies of smooth, or sparsely spined, nonpyramidal cells have been described in the barrels of rats and mice and in layer IV of other species and cortices as well (see Simons and Woolsey, 1984). For example, multipolar and bitufted smooth barrel cells have been reported to receive very different proportions of their excitatory synapses from the thalamus (White et al., 1984). Subclasses of spiny and smooth barrel cells were not modeled explicitly here. Nevertheless, we did purposely introduce some heterogeneity within the two cell populations by allowing synaptic connectivities and weights to vary according to a Gaussian distribution. Other distributions might be more realistic. As noted in the Results, Figure 4 shows evidence for distinct FSU subpopulations that may differ according to the number or effectiveness of inhibitory synapses on them (see McCormick et al., 1985).

$A$ network of cortical cells. A major assumption of our model is that barrel neurons are synaptically interconnected. Although there is presently no direct evidence for intrabarrel connections, the existence of a neuronal network in real barrels is strongly supported by a variety of anatomical studies (see introductory remarks). We have assumed that a majority of the synaptic connections in a barrel originate from neurons within it. Some proportion of a barrel's nonthalamic synapses are undoubtedly of extra-barrel origin (White and Keller, 1987; Armstrong-James et al., 1991). Our model ignored potential contributions to barrel function of these latter inputs.

Synaptic populations within the model barrel were determined in part by our choice of a network size of 100 neurons and the assignment of $30 \%$ of them as inhibitory. Cell connectivities were further constrained by our initial selection of TC convergence factors and our estimates of synapse populations in real barrels that were based on serial reconstructions of a small number of neurons by White and colleagues (see Materials and Methods). One consequence of the methodology is that spiny cells were assigned greater divergences than smooth cells. Light microscopic studies of Golgi-impregnated barrel neurons indicate that smooth cells have longer axons and more branch points than spiny cell, suggesting that the former may have a greater divergence than the latter (Harris and Woolsey, 1983). This inconsistency stems in part from the technical difficulties in the biological studies from which estimates of cell numbers and synaptic populations are derived and from our assumptions regarding barrel circuitry, including the simplifying restriction that possible multiple synaptic contacts from one cell to another are represented by a single synapse. Nevertheless, the transfer functions from real and simulated data in Figure 7 indicate that inhibitory barrel cells do in fact have an effect consistent with an extensive local divergence. In the model barrel, this effect was accomplished by making the smooth cell outgoing synaptic weights larger than those of spiny cells and by having IPSPs decay more slowly than EPSPs.

A second consequence of our procedures for determining synaptic populations within the model barrel is that a given spiny or smooth cell could directly influence a large proportion of the total number of neurons in the network. Each modeled spiny cell could contact as many as $90 \%(60 \pm 30)$ of all barrel neurons, and a smooth cell could contact as many as 60\% (40 \pm 20$)$. Any use of a scalcd-down network in simulating a large neuronal assembly forces a tradeoff between having a realistic proportional interconnectedness and realistic numbers of synaptic inputs to individual neurons; if one opts for having a realistic proportional interconnectedness, then the number of synaptic contacts per cell may be too small to provide adequate spatial integration (MacGregor and Tajchman, 1988). We found that good output could be oblained with networks having fine but not coarse grains, and thus, from an integrative view, the network's extensive interconnectedness errs on the conservative side. The extent to which the interconnectedness within the model barrel mimics that within real barrels is difficult to assess. We predict, however, that virtually identical results could be readily obtained with a much larger, more numerically realistic network wherein the interconnectedness could be proportionately decreased without changing the grain. We have also not 
explored possible effects of structure within the barrel such as specificity of connections among cells having particular functional attributes, for example, directional preferences, or the existence of gradients of local connectivities, that is, near-neighborness. In this regard, a single barrel may consist of several densely interconnected subnetworks that are themselves interconnected to varying degrees. Anatomical and physiological evidence suggests that a barrel's organization may in fact be heterogeneous (see Chmielowska et al., 1989).

Thalamocortical relations. The model was constructed under the assumption that a given barrel cell receives input from more than one neuron in its homologous thalamic barreloid (see introductory remarks). Simulation results indicated that, to produce appropriate spontaneous activity levels and ON and OFF responses, the model required some TC convergence. Such convergence is thought to underlie the broader angular tuning of barrel neurons relative to their thalamic inputs. In our simulations the thalamic data were adjusted so that all of the network's inputs were from cells that responded best to the same direction (upward) of whisker movement, and the simulated barrel neurons were more selective for stimulus direction than their real counterparts. This finding suggests that real TC connections may be less stringently specified than modeled here. As is the case for real barrel cells, however, the model spiny cells were less broadly tuned for deflection angle than smooth cells even though in the model they received slightly more VB inputs (see Table 1). This parallels the disproportionate responsiveness of spiny cells to stimulus onsets versus offsets and to PW versus adjacent whisker stimulation; in this case the strongest input levels occurred with upward deflection angles. Accordingly, microiontophoretic application of GABA antagonists onto an RSU should, by rendering it more responsive, concurrently decrease its $\mathrm{ON}$-to-OFF ratio, increase its receptive field size, and reduce its angular tuning.

We found that good overall output could be achieved over fairly broad ranges of synaptic drives, provided that the ratios of total excitation to total inhibition, and extrinsic to intrinsic excitation, remained approximately constant (see Fig. 8). While we do not presently attach any significance to the ratio values indicated by the model, it seems reasonable that neuronal networks serving different information processing roles may be characterized by different ratios and that such ratios may be experimentally determinable. A general conclusion from our analyses is that a balance is required between how much the model network's behavior depends on its input and how much the network determines its own behavior. Whisking behavior, like other forms of active touch, is dynamic (Carvcll and Simons, 1990). Perhaps the repetitive scanning of a textured surface by the whiskers is associated with transient changes in barrel circuitry (see Juliano and Whitsel, 1987; Lee and Whitsel, 1992). A selective modulation of thalamic or cortical synaptic efficacy would alter tension in the network and thereby enhance or diminish certain aspects of the barrel's response to a vibrissa's displacement. For example, increasing spiny cell tension, by increasing the weight of their divergent synapses, further enhances the disproportionate transformation of strong versus weak responses, as evidenced by increases in the simulated ON-toOFF ratios.

An important advance in the understanding of cortical circuits was the finding (White, 1978, 1979) that TC axons synapse onto smooth cells and do so in a fashion that may enable the thalamus to exert stronger influence on them than on spiny cells (Rall,
1967; Tsukahara et al., 1975). Strong inhibition can be evoked by feedforward, as well as by feedback, circuitry, and the activation of smooth neurons does not depend on concurrent activation of spiny neurons; that is, responses of inhibitory cells are somewhat independent of those of excitatory neurons. Thus, adjacent whisker stimuli evoke vigorous excitation in smooth but not spiny cells, creating strong inhibitory surrounds in the latter. Such a role for smooth cells was demonstrated by the disappearance of cross-whisker inhibition when all network interconnections were removed. Perhaps a more interesting finding in this case was the increase in the spiny cell excitatory response evoked by adjacent whisker deflections, despite the absence of all intrabarrel excitatory connections and adjustment of parameter values to maintain $\mathrm{PW}$ responses within their appropriate ranges. We interpret these results to mean that the high spontaneous activities of smooth cells, which depend in large part on thalamic inputs, generate tonic inhibition that works in conjunction with neuronal threshold and network recurrent excitation nonlinearities to diminish further the relatively weak responses of spiny cells to adjacent whisker deflections. Changes in the spontaneous activities of thalamic neurons could therefore affect receptive field sizes in the cortex. Such changes in the thalamus could underlie, at least in part, the immediate and transient expansion of cortical receptive fields associated with focal injections of anesthetic into the skin (Calford and Tweedale, 1991). Thus, thalamic relay neurons not only provide essential drive to the cortex but can also directly regulate how the cortex responds to it.

\section{References}

Agmon A, Connors BW (1992) Correlation between intrinsic firing patterns and thalamocortical synaptic responses of neurons in mouse barrel cortex. J Neurosci 12:319-329.

Armstrong-James M, Callahan CA, Friedman MA (1991) Thalamocortical processing of vibrissal information in the rat. I. Intracortical origins of surround but not centre-receptive fields of layer IV neurones in the rat S1 barrel field cortex. J Comp Neurol 303:193-210.

Benshalom G, White EL (1986) Quantification of thalamocortical synapses with spiny stellate neurons in layer IV of mouse somatosensory cortex. J Comp Neurol 253:303-314.

Bernardo KL, Woolsey TA (1987) Axonal trajectories between mouse somatosensory thalamus and cortex. J Comp Neurol 258:542-564.

Bernardo KL, McCasland JS, Woolsey TA (1990a) Local axonal trajectories in mouse barrel cortex. Exp Brain Res 82:247-253.

Bcrnardo KL, McCasland JS, Woolscy TA, Strominger RN (1990b) Local intra- and interlaminar connections in mouse barrel cortex. $\mathrm{J}$ Comp Neurol 291:231-255.

Calford MB, Tweedale $R$ (1991) Immediate expansion of receptive fields of neurons in area $3 \mathrm{~b}$ of macaque monkeys after digit denervation. Somatosens Motor Res 8:249-260.

Carvell GE, Simons DJ (1988) Membrane potential changes in rat SmI cortical neurons evoked by controlled stimulation of mystacial vibrissae. Brain Res 448:186-191.

Carvell GE, Simons DJ (1990) Biometric analyses of vibrissal tactile discrimination in the rat. J Neurosci 10:2638-2648.

Chmielowska J, Carvell GE, Simons DJ (1989) Spatial organization of thalamocortical and corticothalamic projection systems in the rat SmI barrel cortex. J Comp Neurol 285:325-338.

Curcio CA, Colcman PD (1982) Stability of neuron number in cortical barrels of aging mice. J Comp Neurol 212:158-172.

Harris RM, Woolsey TA (1983) Computer-assisted analyses of barrel neuron axons and their putative synaptic contacts. $J$ Comp Neurol 220:63-79.

Jensen KF, Killackey HP (1987) Terminal arbors of axons projecting to the somatosensory cortex of the adult rat. I. The normal morphology of specific thalamocortical afferents. J Neurosci 7:3529-3542.

Johannesma PIM, Van den Boogaard HFP (1985) Stochastic formulation of neural interaction. Acta Appl Math 4:201-224.

Juliano SL, Whitsel BL (1987) A combined 2-deoxyglucose and neu- 
rophysiological study of primate somatosensory cortex. J Comp Neurol 263:514-525.

Keller A, White EL (1987) Synaptic organization of GABAergic neurons in mouse SmI cortex. J Comp Neurol 262:1-12.

Koch C, Poggio T, Torre V (1983) Non-linear interactions in a dendritic tree: localization, timing and role in information processing. Proc Natl Acad Sci USA 80:2799-2802.

Koralek K, Jensen KF, Killackey HP (1988) Evidence for two complementary patterns of thalamic input to the rat somatosensory cortex. Brain Res 463:346-351

Land PW, Simons DJ (1985) Cytochrome oxidase staining in the rat SmI barrel cortex. J Comp Neurol 238:225-235.

Land PW, Simons DJ, Buffer SA (1986) Specificity of thalamocortical connections in the rat somatosensory system. Soc Neurosci Abstr 12 1434.

Lee C-J, Whitsel BL (1992) Mechanisms underlying somatosensory cortical dynamics. I. In vivo studies. Cereb Cortex 2:81-106.

Lee KJ, Woolsey TA (1975) A proportional relationship between peripheral innervation density and cortical neuron number in the somatosensory system of the mouse. Brain Res 99:349-353.

Lichtenstein SH, Carvell GE, Simons DJ (1990) Responses of rat trigeminal ganglion neurons to movements of vibrissae in different directions. Somatosens Motor Res 7:47-65.

Lin C-S, Lu SM, Schmechel DE (1985) Glutamic acid decarboxylase activity in layer IV of barrel cortex of rat and mouse. J Neurosci 5:1934-1939.

MacGregor RJ, Tajchman G (1988) Theory of dynamic similarity in neuronal systems. J Neurophysiol 60:751-768.

Mason A, Nicoll A, Stratford K (1991) Synaptic transmission between individual pyramidal neurons of the rat visual cortex in vitro. J Neurosci 11:72-84.

McCormick DA, Connors BW, Lighthall JW, Prince DA (1985) Comparative electrophysiology of pyramidal and sparsely spiny stellate neurons of the neocortex. J Neurophysiol 54:782-806.

Mountcastle VB (1979) An organizing principle for cerebral function: the unit module and the distributed system. In: The neurosciences: fourth study program (Schmitt FO, Worden FG, eds), pp 21-42. Cambridge, MA: MIT Press.

Pasternak JF, Woolsey TA (1975) The number, size and spatial distribution of neurons in lamina IV of the mouse SmI neocortex. J Comp Neurol 160:291-306.

Rall W (1967) Distinguishing theoretical synaptic potentials computed for different soma-dendritic distributions of synaptic input. J Neurophysiol 30:1138-1168.

Rumelhart DE, Hinton GE, McClelland JL (1986) A general framework for parallel distributed processing. In: Parallel distributed processing: explorations in the microstructure of cognition, Vol 1, Foundations (Rumelhart DE, McClelland JL, eds), pp 45-76. Cambridge, MA: MIT Press.
Shepherd GM, Crayton RK, Miller JP, Segev I, Rinzel J, Rall W (1985) Signal enhancement in distal cortical dendrites by means of interactions between active dendritic spines. Proc Natl Acad Sci USA 82: 2192-2195.

Simons DJ (1978) Response properties of vibrissa units in the rat SI somatosensory neocortex. J Neurophysiol 41:798-820.

Simons DJ (1985) Temporal and spatial integration in the rat SI vibrissa cortex. J Neurophysiol 54:615-635.

Simons DJ, Carvell GE (1989) Thalamocortical response transformation in the rat vibrissa/barrel system. J Neurophysiol 61:311-330.

Simons DJ, Woolsey TA (1984) Morphology of Golgi-Cox-impregnated barrel neurons in rat SmI cortex. J Comp Neurol 230:119-132.

Spreafico R, DeBiasi S, Frassoni C, Battaglia G (1988) A comparison of GAD- and GABA-immunoreactive neurons in the first somatosensory area (SI) of the rat cortex. Brain Res 474:192-196.

Swadlow HA (1989) Efrerenl neurons and suspected interneurons in S-1 vibrissa cortex of the awake rabbit: receptive fields and axonal properties. J Neurophysiol 62:288-308.

Tsukahara N, Murakami F, Hultborn H (1975) Electrical constants of neurons of the red nucleus. Exp Brain Res 23:49-64.

Welker C, Woolsey TA (1974) Structure of layer IV in the somatosensory neocortex of the rat: description and comparison with the mouse. J Comp Neurol 158:437-453.

White EL (1978) Identified neurons in mouse SmI cortex which are postsynaptic to thalamocortical axon terminals: a combined Golgielectron microscopic and degeneration study. J Comp Neurol 181: 627-661.

White EL (1979) Thalamocortical synaptic relations: a review with emphasis on projections of specific thalamic nuclei to the primary sensory areas of the neocorlex. Brain Res Rev 1:275-311.

White EL (1989) Cortical circuits: synaptic organization of the cerebral cortex. Boston: Birkhauser.

White EL, Keller A (1987) Intrinsic circuitry involving the local axon collaterals of corticothalamic projection cells in mouse SmI cortex. J Comp Neurol 262:13-26.

White EL, Rock MP (1980) Three-dimensional aspects and synaptic relationships of a Golgi-impregnated spiny stellate cell reconstructed from serial thin sections. J Neurocytol 9:615-636.

White EL, Benshalom G, Hersch SM (1984) Thalamocortical and other synapses involving nonspiny multipolar cells of mouse SmI cortex. J Comp Neurol 229:311-320.

Wilson SJ (1988) Cellular mechanisms controlling the strength of synapses. J Electron Microsc Tech 10:293-313.

Woolsey TA, Van der Loos H (1970) The structural organization of layer IV in the somatosensory region (SI) of mouse cerebral cortex. Brain Res 17:205-242.

Woolsey TA, Dierker ML, Wann DF (1975) Mouse Sml cortex: qualitative and quantitative classification of Golgi-impregnated barrel neurons. Proc Natl Acad Sci USA 72:2165-2169. 